\title{
A finite volume-based high-order, Cartesian cut-cell method for wave propagation
}

\author{
Mihaela Popescu ${ }^{1}$, Rick Vedder ${ }^{2}$ and Wei Shyy ${ }^{3, *, \dagger}$ \\ ${ }^{1}$ Department of Process Technology, Flow Technology Group, SINTEF Materials and Chemistry, \\ Trondheim, Norway \\ ${ }^{2}$ Department of Electrical Engineering, Mathematics and Computer Science, Delft University of Technology, \\ Delft, The Netherlands \\ ${ }^{3}$ Department of Aerospace Engineering, University of Michigan, Ann Arbor, MI 48109, U.S.A.
}

\begin{abstract}
SUMMARY
Computational aeroacoustics requires numerical techniques capable of yielding low artificial dispersion and dissipation to preserve the amplitude and the frequency characteristics of the physical processes. Furthermore, for engineering applications, the techniques need to handle irregular geometries associated with realistic configurations. We address these issues by developing an optimized prefactored compact finite volume (OPC-fv) scheme along with a Cartesian cut-cell technique. The OPC-fv scheme seeks to minimize numerical dispersion and dissipation while satisfying the conservation laws. The cut-cell approach treats irregularly shaped boundaries using divide-and-merge procedures for the Cartesian cells while maintaining a desirable level of accuracy. We assess these techniques using several canonical test problems, involving different levels of physical and geometric complexities. Richardson extrapolation is an effective tool for evaluating solutions of no high gradients or discontinuities, and is used to evaluate the performance of the solution technique. It is demonstrated that while the cut-cell method has a modest effect on the order of accuracy, it is a robust method. The combined OPC-fv scheme and the Cartesian cut-cell technique offer good accuracy as well as geometric flexibility. Copyright (c) 2008 John Wiley \& Sons, Ltd.
\end{abstract}

Received 12 October 2006; Revised 21 March 2007; Accepted 26 March 2007

KEY WORDS: cut cell; Richardson extrapolation; aeroacoustic; OPC-fv

\section{INTRODUCTION}

In computational aeroacoustics (CAA), accurate prediction of sound generation is challenging due to the requirement for preservation of both the amplitude and frequency contents of the wave

\footnotetext{
${ }^{*}$ Correspondence to: Wei Shyy, Department of Aerospace Engineering, University of Michigan, Ann Arbor, MI 48109, U.S.A.

†E-mail: weishyy@umich.edu

Contract/grant sponsor: NASA Constellation University Institutes Program (CUIP)
}

Copyright (C) 2008 John Wiley \& Sons, Ltd. 
generation and propagation. Furthermore, suitable numerical schemes need to handle multiple scales, including long and short wavelengths, as well as nonlinear governing laws arising from sources, such as turbulence, shocks, interaction between fluid flows and elastic structures, and complex geometries. It is well recognized (e.g. [1,2]) that in order to conduct satisfactory CAA simulations, numerical schemes need to minimize dispersion and dissipation errors. In general, higher-order schemes are more suitable for CAA because they are less dissipative than lower-order schemes [3-5].

For waves with long wavelengths relative to the grid size, the formal order of accuracy is sufficient to indicate the performance of a scheme. However, for waves whose wavelengths are shorter than or comparable to the grid size, it is known that the leading truncation error terms are not good indicators $[6,7]$. To handle broadband waves, the idea of optimizing scheme coefficients by minimizing the truncation error associated with a particular range of wavenumbers has been adopted by many researchers (e.g. [8-15]). As demonstrated by Tam et al. [2], one can optimize

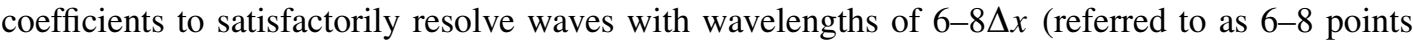
per wave) or shorter. The resulting scheme optimizes the accuracy by simultaneously handling the wavenumber and frequency characteristics in the range of resolvable scales. Ashcroft and Zhang [14] have reported a strategy for developing optimized prefactored compact (OPC) schemes, which splits the central implicit schemes into forward- and backward-biased operators, subject to a measure of optimality.

The OPC scheme was originally designed based on a finite difference approach. In order to satisfy the governing physical laws, it can be advantageous to adopt the finite volume approach, which ensures that the fluxes estimated from different sides of the same surface to be identical. In other words, no spurious sources or sinks are generated due to numerical treatment. Such a requirement can be important when nonlinearity is involved. Furthermore, a finite volume formulation can offer an orderly framework to handle the irregular geometries and moving boundaries. Popescu et al. [15] have extended the finite difference schemes originally proposed by Tam et al. [2] and by Ashcroft and Zhang [14] to suitable finite volume forms, and offered systematic assessment of their performance.

In addition to handling the fundamental characteristics of waves, geometric complexity is another critical issue. Since practical engineering problems often involve irregularly shaped geometries, suitable techniques need to be employed in order to maintain the accuracy of high-fidelity computations in such configurations. In this study, a Cartesian cut-cell approach based on the OPC finite volume (OPC-fv) scheme, aimed at minimizing dispersion and dissipation while offering geometric flexibility, is proposed and evaluated. This approach has the merit that the burden on grid generation is lessened and the accuracy of the overall computations between the equation solver and the geometric resolution are coordinated through the numerical solution techniques. Specifically, the governing equations in Cartesian coordinates are solved using the OPC-fv scheme [15]. The low-dispersion and low-dissipation Runge-Kutta (LDDRK) by Hu [16] will be used for time discretization. For the treatment of boundary condition, we propose a modified perfect matched layer (PML), which fits better to Navier-Stokes equations. The approach follows the one developed by $\mathrm{Hu}$ [17], who has offered substantial details.

To assess the performance of the resulting numerical technique, we will compute the wave radiation produced by oscillating baffled pistons using both linearized Euler equations and nonlinear Navier-Stokes equations. Wave generation from a vibrating circular piston is a classical acoustic problem, and has been investigated by, among others, Freedmann [18], Lele and Hamilton [19], Cleveland et al. [20], Williams [21], Blackstock [22], and Cheong and Lee [12]. 
In practical applications, not only a single wave radiated from an isolated baffled piston, but also the interaction between waves from an array of baffled pistons, and radiated wave around complex geometries are of interest. In order to solve such problems, we will study the wave radiation from baffled pistons and their interaction with irregular geometries or the interaction from multiple pistons located at different points in the two-dimensional space. The challenge in this computation is to capture the characteristics of the waves initiated by the pistons, and wavesolid wall and wave-wave interactions, in terms of frequency, amplitude and wavenumber. In order to systematically evaluate the performance of the present numerical schemes, the Richardson extrapolation technique is employed. We will investigate whether Richardson extrapolation is an effective tool to improve numerical solution, or assess the order of accuracy of a numerical scheme for wave computations.

The remaining paper is organized as follows. The governing equations, numerical schemes, and boundary conditions will be discussed briefly in Section 2. In this section, we will also present the cut-cell technique and the Richardson extrapolation approach. In Section 3 we present the results and discussions of the numerical simulation of the baffled pistons for linear and nonlinear cases. The last section will offer conclusion and remarks.

\section{NUMERICAL SCHEMES FOR SPACE AND TIME DISCRETIZATION}

\subsection{Space discretization-optimized prefactored compact finite volume scheme (OPC-fv)}

Consider the first-order, one-dimensional linear wave equation

$$
\frac{\partial u}{\partial t}+c \frac{\partial u}{\partial x}=0
$$

To derive the discretized equation, we employ the grid point cluster shown in Figure 1, focusing on the grid point $i$, who has the grid points $i-1$ and $i+1$ as its neighbors. The dashed lines define the control volume, and the letters $e$ and $w$ denote the east and west faces of the control volume, respectively.

To offer a better understanding of the OPC-fv scheme, we first summarize the original finite difference version of the OPC scheme developed by Ashcroft and Zhang [14], termed OPC-fd. The factorized compact scheme in the finite difference approach is obtained by defining the forward and backward operators $D_{i}^{\mathrm{F}}$ and $D_{i}^{\mathrm{B}}$ such that

$$
\left(\frac{\partial u}{\partial x}\right)_{i}=\frac{1}{2}\left(D_{i}^{\mathrm{B}}+D_{i}^{\mathrm{F}}\right)
$$

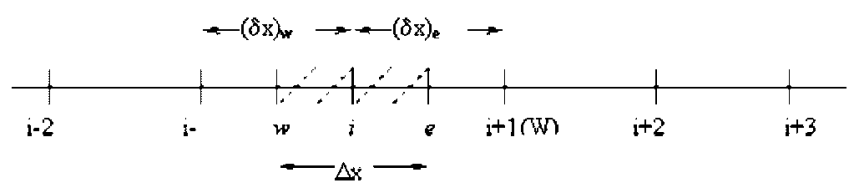

Figure 1. Grid layout and notation for one-dimensional problem. 
The generic stencils for fourth-order forward and backward derivative operators are given by

$$
\eta_{\mathrm{F}} D_{i+1}^{\mathrm{F}}+\beta_{\mathrm{F}} D_{i}^{\mathrm{F}}=\frac{1}{\Delta x}\left[a_{\mathrm{F}} u_{i+2}+b_{\mathrm{F}} u_{i+1}+c_{\mathrm{F}} u_{i}+d_{\mathrm{F}} u_{i-1}+e_{\mathrm{F}} u_{i-2}\right]
$$

and

$$
\beta_{\mathrm{B}} D_{i}^{\mathrm{B}}+\eta_{\mathrm{B}} D_{i-1}^{\mathrm{B}}=\frac{1}{\Delta x}\left[a_{\mathrm{B}} u_{i+2}+b_{\mathrm{B}} u_{i+1}+c_{\mathrm{B}} u_{i}+d_{\mathrm{B}} u_{i-1}+e_{\mathrm{B}} u_{i-2}\right]
$$

The coefficients are obtained by imposing that: (i) the scheme has a certain order of accuracy; and (ii) dispersion and dissipation are minimized over a selected window of frequency. As illustrated in Figure 1, the points $i, i+1$, etc. are the nodes where the dependent variables are defined, while $e$ and $w$ define the boundary of a cell centered at point $i$. The finite volume formulation of the optimized prefactored scheme is obtained by taking into account Equations (2)-(4), using the idea that the approximation of function at points $e$ and $w$ should have similar forms. For simplicity, we consider a one-dimensional problem with unit thickness in the $y$ and $z$ directions

$$
\int_{e}^{w} \frac{\partial u}{\partial t} \mathrm{~d} x+c\left((A u)_{e}-(A u)_{w}\right)=0
$$

where $(A u)_{e}$ and $(A u)_{w}$ are the fluxes across the east and west faces, respectively. Hence, the discretized wave Equation (1) can be written as

$$
\frac{\partial \bar{u}}{\partial t} \Delta x+c\left((A u)_{e}-(A u)_{w}\right)=0
$$

where $\bar{u}$ is the averaged value of $u$ over the control volume.

Based on the OPC-fd scheme, the value of the function in the center of the face is defined by the relations

$$
\begin{aligned}
& u_{e}=0.5\left(u^{\mathrm{F} e}+u^{\mathrm{B} e}\right) \\
& u_{w}=0.5\left(u^{\mathrm{F} w}+u^{\mathrm{B} w}\right)
\end{aligned}
$$

where $u^{\mathrm{F} e}, u^{\mathrm{B} e}, u^{\mathrm{F} w}$, and $u^{\mathrm{B} w}$ are determined from

$$
\begin{aligned}
\eta u_{i+1}^{\mathrm{F} e}+\beta u_{i}^{\mathrm{F} e} & =b u_{i+1}-d u_{i} \\
\eta u_{i+1}^{\mathrm{F} w}+\beta u_{i}^{\mathrm{F} w} & =b u_{i}-d u_{i-1} \\
\beta u_{i}^{\mathrm{B} e}+\eta u_{i-1}^{\mathrm{B} e} & =b u_{i}-d u_{i+1} \\
\beta u_{i}^{\mathrm{B} w}+\eta u_{i-1}^{\mathrm{B} w} & =b u_{i-1}-d u_{i}
\end{aligned}
$$

and the coefficients are the same as those in the OPC-fd scheme (see Equations (3) and (4))

$$
\begin{aligned}
& \eta=\eta_{\mathrm{F}}=\gamma_{\mathrm{B}} \\
& \beta=\beta_{\mathrm{F}}=\beta_{\mathrm{B}} \\
& b=b_{\mathrm{F}}=-d_{\mathrm{B}} \\
& d=d_{\mathrm{F}}=-b_{\mathrm{B}}
\end{aligned}
$$




\subsection{Time discretization-low dispersion and dissipation Runge-Kutta (LDDRK) method}

$\mathrm{Hu}$ et al. [8] considered time integration using the Runge-Kutta algorithm for the differential equation

$$
\frac{\partial u}{\partial t}=F(u)
$$

where the operator $F$ is a function of $u$. An explicit $p$-stage algorithm advances the solution of Equation (10) from the $n$th to the $(n+1)$ th iteration

$$
\begin{aligned}
u^{(0)} & =u^{n} \\
K^{(1)} & =\Delta t F\left(u^{(0)}\right) \\
& \vdots \\
K^{(i)} & =\Delta t F\left(u^{(i-1)}\right) \\
u^{(i)} & =u^{n}+b_{i} K^{(i)}, \quad i=1, \ldots, p \\
& \vdots \\
u^{n+1} & =u^{(p)}
\end{aligned}
$$

where $b_{p}=1$.

The coefficients of the LDDRK are obtained such that: (i) the scheme has a prescribed order of accuracy; (ii) the error of the amplification factor of the scheme over the specified phase range is minimized; and (iii) the amplification factor of the scheme is within the stability limit.

In this work, we use a two-step alternating scheme. In the first step, we use four stages, and in the second we use six stages. The scheme is fourth-order accurate in time for a linear problem and second-order accurate for a nonlinear problem [8].

The specific procedure is given below.

1. Four-stage:

$$
\begin{aligned}
K^{(1)} & =\Delta t F\left(u^{n}\right) \\
K^{(2)} & =\Delta t F\left(u^{n}+\frac{1}{4} K^{(1)}\right) \\
K^{(3)} & =\Delta t F\left(u^{n}+\frac{1}{3} K^{(2)}\right) \\
K^{(4)} & =\Delta t F\left(u^{n}+\frac{1}{2} K^{(3)}\right) \\
u^{n+1} & =u^{n}+K^{(4)}
\end{aligned}
$$

2. Six-stage:

$$
\begin{aligned}
& K^{(1)}=\Delta t F\left(u^{n}\right) \\
& K^{(2)}=\Delta t F\left(u^{n}+0.17667 K^{(1)}\right) \\
& K^{(3)}=\Delta t F\left(u^{n}+0.38904 K^{(2)}\right)
\end{aligned}
$$




$$
\begin{aligned}
& K^{(4)}=\Delta t F\left(u^{n}+\frac{1}{4} K^{(3)}\right) \\
& K^{(5)}=\Delta t F\left(u^{n}+\frac{1}{3} K^{(4)}\right) \\
& K^{(6)}=\Delta t F\left(u^{n}+\frac{1}{2} K^{(5)}\right) \\
& u^{n+1}=u^{n}+K^{(6)}
\end{aligned}
$$

In this study, the LDDRK scheme and the OPC-fv scheme are combined. In the context of the procedures presented above

$$
F_{l}=-c\left(u_{l}^{e}-u_{l}^{w}\right) / \Delta x
$$

where

$$
u_{l}^{e}=0.5\left(u_{l}^{\mathrm{B} e}+u_{l}^{\mathrm{Fe}}\right) \quad \text { and } \quad u_{l}^{w}=0.5\left(u_{l}^{\mathrm{B} w}+u_{l}^{\mathrm{F} w}\right)
$$

\subsection{Boundary treatment}

To handle the outflow boundary for nonlinear case, the PML is adopted. The PML was designed for Euler's equation by $\mathrm{Hu}$ [16]. In order to apply PML to the Navier-Stokes equations, we add the diffusive term into the original PML equations. This approach was also developed with more details by $\mathrm{Hu}$ [17]. The equations that characterize PML are as follows:

$$
\begin{aligned}
& \frac{\partial u_{1}^{x}}{\partial t}+\sigma_{x} u_{1}^{x}=-\frac{1}{\rho} \frac{\partial p}{\partial x}-u^{x} \frac{\partial u^{x}}{\partial x}+\frac{1}{R e} \frac{\partial^{2} u^{x}}{\partial x^{2}} \\
& \frac{\partial u_{2}^{x}}{\partial t}+\sigma_{y} u_{2}^{x}=-u^{y} \frac{\partial u^{x}}{\partial y}+\frac{1}{R e} \frac{\partial^{2} u^{x}}{\partial y^{2}} \\
& \frac{\partial u_{1}^{y}}{\partial t}+\sigma_{x} u_{1}^{y}=-u^{x} \frac{\partial u^{v}}{\partial x}+\frac{1}{R e} \frac{\partial^{2} u^{y}}{\partial x^{2}} \\
& \frac{\partial u_{2}^{y}}{\partial t}+\sigma_{y} u_{2}^{y}=-\frac{1}{\rho} \frac{\partial p}{\partial y}-u^{y} \frac{\partial u^{y}}{\partial y}+\frac{1}{R e} \frac{\partial^{2} u^{y}}{\partial y^{2}} \\
& \frac{\partial p_{1}}{\partial t}+\sigma_{x} p_{1}=-\gamma p \frac{\partial u^{x}}{\partial x}-u^{x} \frac{\partial p}{\partial x} \\
& \frac{\partial p_{2}}{\partial t}+\sigma_{y} p_{2}=-\gamma p \frac{\partial u^{y}}{\partial y}-u^{y} \frac{\partial p}{\partial y} \\
& \frac{\partial \rho_{1}}{\partial t}+\sigma_{x} \rho_{1}=-\frac{\partial\left(\rho u^{x}\right)}{\partial x} \\
& \frac{\partial \rho_{2}}{\partial t}+\sigma_{y} \rho_{2}=-\frac{\partial\left(\rho u^{y}\right)}{\partial y}
\end{aligned}
$$




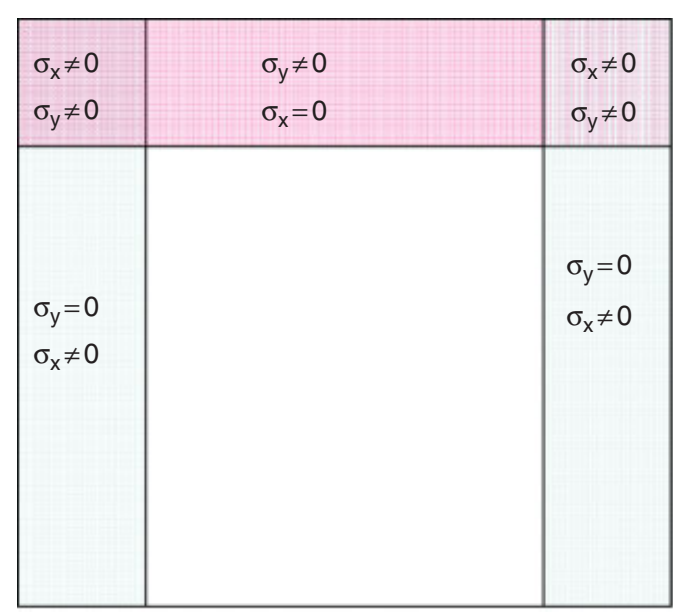

Figure 2. Illustration of computational domain with perfect matched layers as outflow boundary.

where $u^{x}, u^{y}$ indicate velocity in the $x$ and $y$ directions, and $\sigma_{x}$ and $\sigma_{y}$ are defined by the formula:

$$
\sigma=\sigma_{m}\left(\frac{d}{D}\right)^{\beta}
$$

where $\sigma_{m}$ is the maximum value of $\sigma$ (the absorbing coefficient for PML domain), $D$ the width of the PML domain, $d$ the distance from its interface to the interior domain (see Figure 2). In our approach, the PML employs the outflow boundary designed by Tam and Webb [23]:

$$
\frac{\partial p_{i}}{\partial t}+\cos (\theta) \frac{\partial p_{i}}{\partial x}+\sin (\theta) \frac{\partial p_{i}}{\partial y}+\frac{p_{i}}{2 R}=0
$$

where $i=1,2$. In our computation, we used 21 points in PML.

\subsection{The cut-cell procedure for irregular geometry}

The cut-cell method rearranges the computational cells in the vicinity of an interface via subdivision to conform to the specified boundary shape. Depending on the intersection between the grid line and the boundary, the subdivided, or cut, cells can remain independent or can be merged into a neighboring cell in a given direction, e.g. a direction approximately normal to the solid face as illustrated in Figures 3 and 4 (e.g. [24-29]). Accordingly, the boundary cells are reorganized along with their neighboring cells to form new cells with triangular, trapezoidal, or pentagonal shapes.

The flux across the cell boundaries can be approximated by

$$
\oint \mathbf{f} \cdot \mathbf{n} \mathrm{d} s \cong \sum_{k=1}^{m} f_{k} n_{k}
$$

The flux on the cell face is computed based on the multi-dimensional interpolation method [29]. Since the LDDRK and OPC-fv schemes considered here are fourth-order accurate, it is desirable to preserve the same order of accuracy around the boundary. 

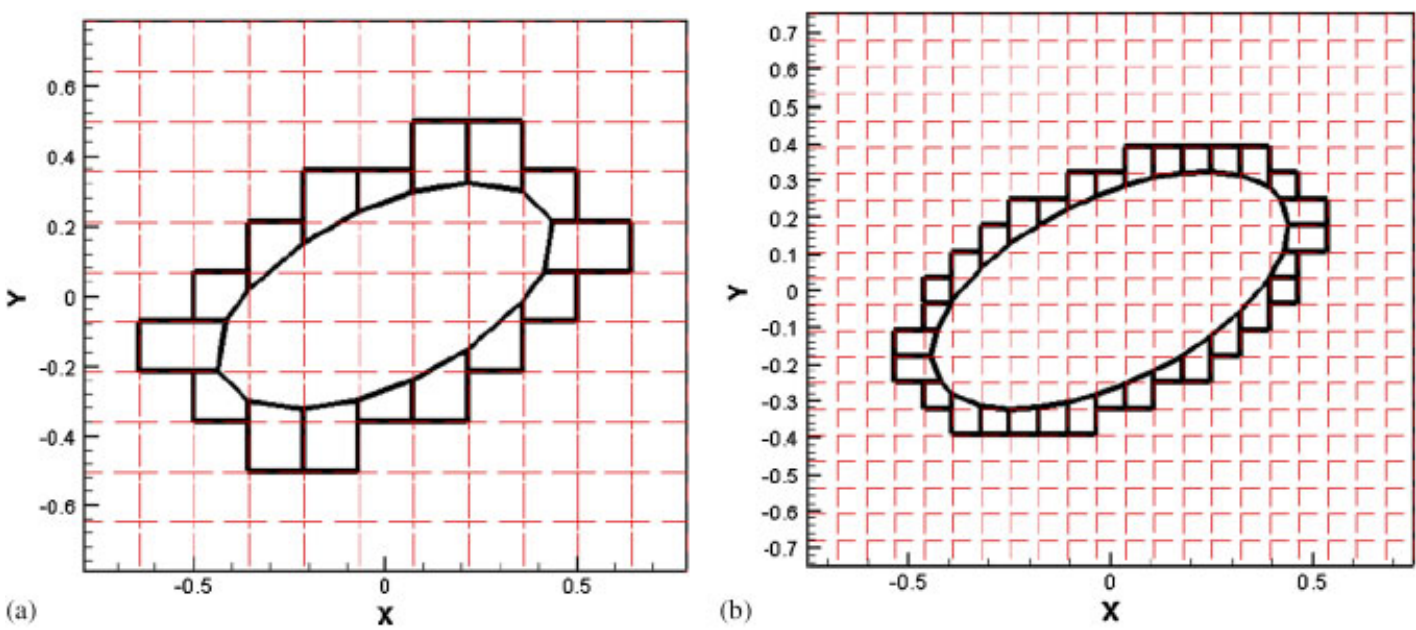

Figure 3. Example of the Cartesian cut-cell method, which results in mixed structure and unstructured grid: (a) coarse grid and (b) finer grid.

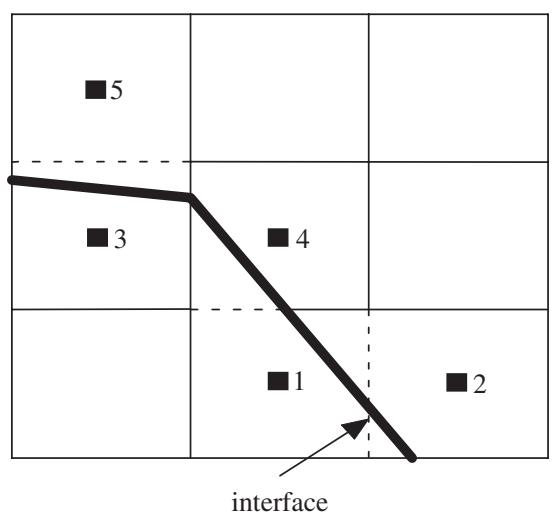

(a)

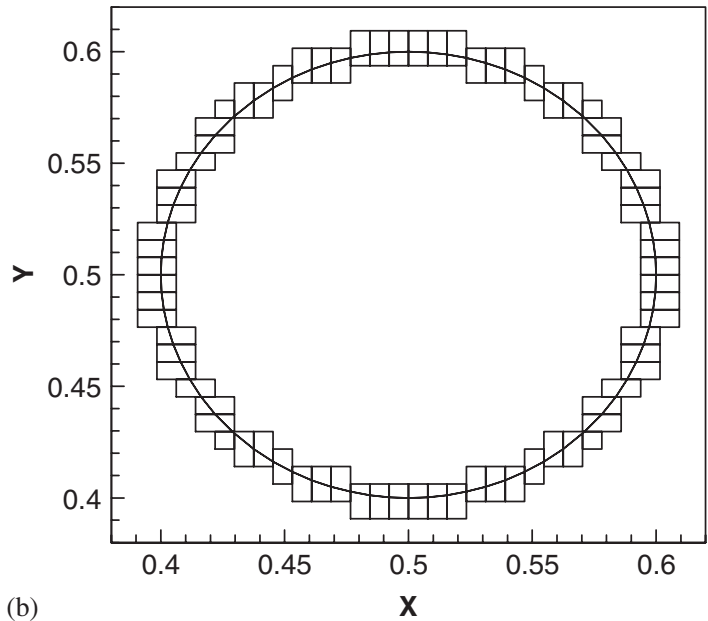

Figure 4. Illustration of the interfacial cells and cut-and-absorption procedures: (a) local situation and (b) cut cells along the interface.

The Cartesian cut-cell approach employs the following steps:

- Locate the intersection of the boundary and the underlying Cartesian mesh.

- Establish the identity of each Cartesian cell. The cells are flagged as solid cells, flow cells, or boundary cells. The boundary cells are those that either intersect the boundary or have a face in common with the boundary.

- Determine the geometric characteristics of the boundary cells, such as cell volume, the direction normal to the boundary, and other information. 


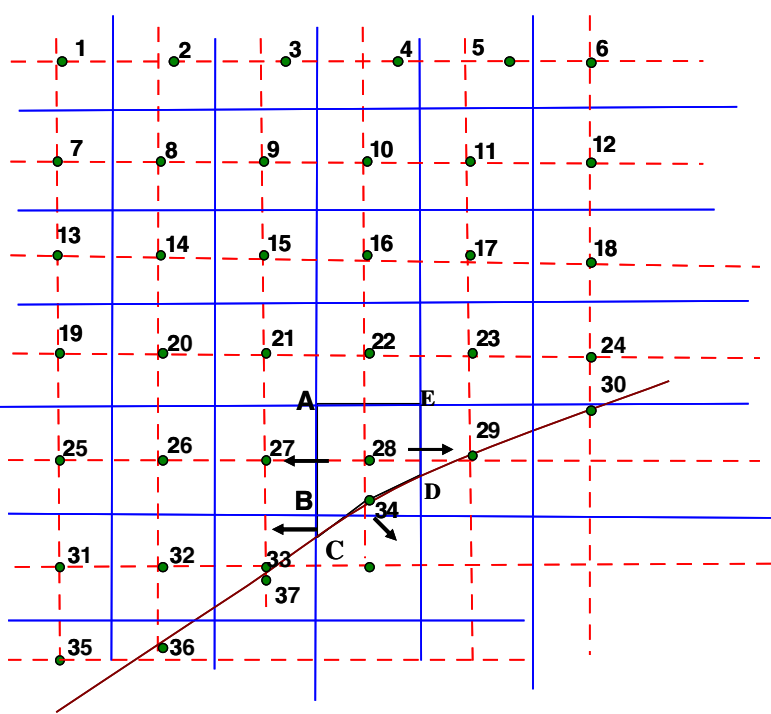

(a)

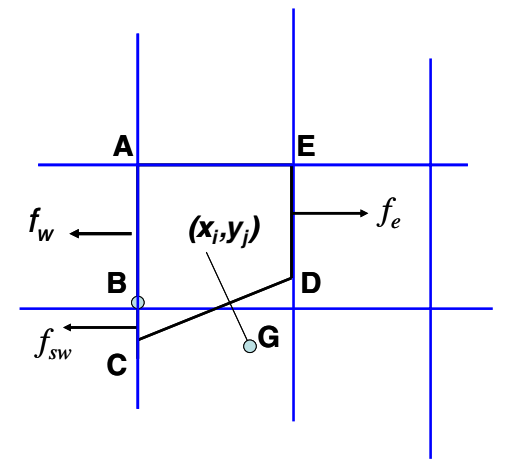

(b)

Figure 5. Modified cut-cell approach for CAA: (a) Cartesian cut-cell approach and (b) detail around the cut cell.

- Merge cells as necessary. A minimum acceptable cell area $S_{\min }$ is specified, and when the area of a cut cell is smaller than this value, it is merged into a neighboring cell. The choice for $S_{\min }$ is based on a trade-off between the time step and resolution accuracy.

- Determine the new characteristics of the merged cells.

For the trapezoid $A C D E$ shown in Figure 5, the finite volume approach can be used to approximate the wave equation

$$
\int_{A B C D E}\left(\frac{\partial u}{\partial t}+\frac{\partial E}{\partial x}+\frac{\partial F}{\partial y}\right) d \forall=0
$$

where $\mathrm{d} \forall$ is a volume element. Applying Stoke's theorem to Equation (20), we have

$$
\frac{\partial u_{c}}{\partial t} S_{A B C D E}+\oint_{A B C D E}(E \mathrm{~d} y-F \mathrm{~d} x)=0
$$

The value of the integral can be approximated by

$$
\oint_{A B C D E}(E \mathrm{~d} y-F \mathrm{~d} x)=\int_{A C} E \mathrm{~d} y+\int_{C D}(E \mathrm{~d} y-F \mathrm{~d} x)+\int_{D E} E \mathrm{~d} y-\int_{E A} F \mathrm{~d} x
$$


The functions $F$ and $E$ can be represented generically by a function $f$. The fluxes on the faces $A C$ and $D E$ can be approximated by

$$
\int_{A C} f \mathrm{~d} y=\int_{A B} f \mathrm{~d} y+\int_{B C} f \mathrm{~d} y
$$

An approximation of the value of the flux is given by

$$
\int_{A C} f \mathrm{~d} y \approx f_{w}\left(y_{A}-y_{B}\right)+f_{s w}\left(y_{B}-y_{C}\right)
$$

The value of the flux at point $w$ is given by the specific formula for the boundary cell. The value of $f_{s w}$ is approximated using a fourth-order polynomial in the $x$ and $y$ directions

$$
f_{s w}=\sum_{i=0}^{4} \sum_{j=0}^{4} b_{i j} x^{i} y^{j}
$$

where the coefficients $b_{i j}$ are unknown. This interpolation has fourth-order accuracy in the evaluation of the flux on the cut cells. In this case, the value of the coefficients is obtained using the values of $f$ at 25 grid points. An example is given in Figure 5 where the value of the function is approximated using 25 points.

To solve for $b_{i j}$, we use the following system of equations by expressing the function $f$ at 25 locations:

$$
\left\{\begin{array}{c}
f_{1} \\
f_{2} \\
\vdots \\
f_{25}
\end{array}\right\}=\left\{\begin{array}{ccccc}
x_{1}^{4} y_{1}^{4} & x_{1}^{3} y_{1}^{4} & x_{1}^{4} y_{1}^{3} \cdots x_{1} & y_{1} & 1 \\
x_{2}^{4} y_{2}^{4} & x_{2}^{3} y_{2}^{4} & x_{2}^{4} y_{2}^{3} \cdots x_{2} & y_{2} & 1 \\
\vdots & \vdots & \vdots & \vdots & \vdots \\
x_{25}^{4} y_{25}^{4} & x_{25}^{3} y_{25}^{4} & x_{25}^{4} y_{25}^{3} \cdots x_{25} & y_{25} & 1
\end{array}\right\}\left\{\begin{array}{c}
b_{1} \\
b_{2} \\
\vdots \\
b_{25}
\end{array}\right\}
$$

The coefficients $b_{i j}$ from Equation (25) become the coefficients $b_{1}, b_{2}, \ldots, b_{25}$ in Equation (26). These coefficients can now be expressed in terms of values of $f$ at the 25 points by inverting Equation (26), i.e.

$$
b_{n}=\sum_{j=1}^{25} a_{n j} f_{j}, \quad n=1, \ldots, 25
$$

where $a_{n j}$ are the elements of the inverse of the matrix in Equation (26).

After $b_{i}$ is obtained, the value of $f$ at the center of $B C$ is expressed in the form

$$
f_{s w}=b_{1} x_{s w}^{4} y_{s w}^{4}+b_{2} x_{s w}^{3} y_{s w}^{4}+b_{3} x_{s w}^{4} y_{s w}^{3}+\cdots+b_{23} x_{s w}+b_{24} y_{s w}+b_{25}
$$

and using Equation (27), the value of $f_{s w}$ can be rewritten as

$$
f_{s w}=\sum_{j=1}^{25} \alpha_{j} f_{j}
$$

Note that $b_{i}$ are coefficients that depend only on the mesh, the location, and the orientation of the boundary. Therefore, with a fixed geometry, these coefficients can be computed once at the beginning of the solution procedure. 
Now we turn our attention to the calculation of the flux on the immersed face $C D$ of the cell $(i, j)$. To compute the flux on a solid boundary, we use the reflection boundary condition. In Figure 5(b), $\left(x_{i}, y_{j}\right)$ is the mass center of the boundary cell. We introduce a point across the boundary that is symmetrical to the center of mass of face $C D$, and denote it as $G$. The variable values at the ghost point $G$ are

$$
\begin{aligned}
p_{G} & =p_{i j} \\
\mathbf{u}_{G} & =\mathbf{u}_{i j}-2\left(\mathbf{u}_{i j} \cdot \mathbf{n}\right) \mathbf{n}
\end{aligned}
$$

where $\mathbf{n}$ is the normal vector of the solid boundary, the subscripts $i j$ indicate the value of variables at point $\left(x_{i}, y_{j}\right)$, and the subscript $G$ indicates the value at a ghost point $G$. The value of the flux on face $C D$ is approximated by

$$
\begin{aligned}
& p_{C D}=\frac{p_{G}+p_{i j}}{2} \\
& \mathbf{u}_{C D}=\frac{\mathbf{u}_{G}+\mathbf{u}_{i j}}{2}
\end{aligned}
$$

Following simple algebra, we can observe that Equation (31) assures that the velocity is zero in the normal direction, and the variation of pressure in the normal direction around the boundary is zero.

\subsection{Richardson extrapolation}

Richardson extrapolation has attracted interests in the CFD community because of its potential to improve the quantitative accuracy as well as the order of accuracy of a given computational technique. It is built upon the concept that by combining two separate discrete solutions, on two different grids, the leading order error term in the assumed error expansion can be eliminated. The extrapolation must be used with considerable caution, since it involves the additional assumption of monotone truncation error convergence in the mesh spacing. The method has a major attractive feature: it is oblivious to the equations being discretized and to the dimensionality of the problem and can easily be applied as a postprocessor to solutions on two grids with no reference to the codes, algorithms, or governing equations that produced the solutions. For the case in which the order of accuracy of the spatial discretization is $p_{1}$ and the order of accuracy of the time integration is $q_{1}$, the difference between the analytical solution $P^{n}$ and the numerical solution $P^{n}(\Delta x, \Delta t)$ with grid spacing $\Delta x$ and time step $\Delta t$ in the $n$th iteration can be written as follows:

$$
P^{n}-P^{n}(\Delta x, \Delta t)=c_{1 x} \Delta x^{p_{1}}+c_{2 x} \Delta x^{p_{1}+1}+\cdots+c_{1 t} \Delta t^{q_{1}}+c_{1 t} \Delta t^{q_{1}+1}+\cdots
$$

where $c_{1 x}, c_{2 x}, \ldots, c_{1 t}, c_{2 t}, \ldots \in \Re$. This can be carried out for every grid point.

For simplicity, consider $\Delta x=\Delta y$, and substitute $\Delta t$ with the Courant-Friedrichs-Lewy (CFL)condition $(\mathrm{CFL}=c \Delta t / \min (\Delta x, \Delta y))$. Keeping the CFL-number constant, Equation (32) can be written as follows:

$$
P^{n}-P^{n}(\Delta x)=C \Delta x^{p}
$$

where $p$ is the order of accuracy and $C \in \Re$. 
In Equation (33) $P^{n}, C$, and $p$ are unknown and can be determined by writing Equation (33) for three solutions with three different grid spacings $\Delta x, \Delta x / 2$, and $\Delta x / 4$. After a mathematical manipulation we obtain

$$
\begin{gathered}
\frac{P^{n}\left(\frac{\Delta x}{2}\right)-P^{n}(\Delta x)}{P^{n}\left(\frac{\Delta x}{4}\right)-P^{n}\left(\frac{\Delta x}{2}\right)}=2^{p} \\
P^{n}=\frac{2^{p} P^{n}\left(\frac{\Delta x}{4}\right)-P^{n}\left(\frac{\Delta x}{2}\right)}{2^{p}-1}
\end{gathered}
$$

From Equation (34) the order of accuracy can be determined in every point of the grid and from Equation (35) an improved extrapolated solution can be determined, which is based on canceling the leading truncation error term in Equation (33).

Richardson extrapolation consists of using three grids of the numerical solutions which will not match, because the solutions are obtained with different grid spacings. Therefore, the numerical solutions have to be interpolated because Richardson extrapolation method can only be applied to solutions on a common grid. This interpolation method should not affect the accuracy of the numerical solution. Therefore, the order of accuracy of the interpolation method must be higher than the derived order of accuracy of the numerical solutions.

Next, we will present the way in which the interpolation is performed in function of the position of the point in the domain.

2.5.1. Interior interpolation. In the interior of the domain, interpolation polynomials of fifth degree in $x$ and $y$ are established by two-dimensional Lagrange polynomials. In order to determine the interpolation polynomial 36 points are needed, which can easily be determined in a $6 \times 6$ square in the interior of the domain. The point to be interpolated is located in the center of this square. In Figure 6 the square is given with the open dots the cell centers, at which the values of the numerical simulations are known, and the closed dot the point to be interpolated.

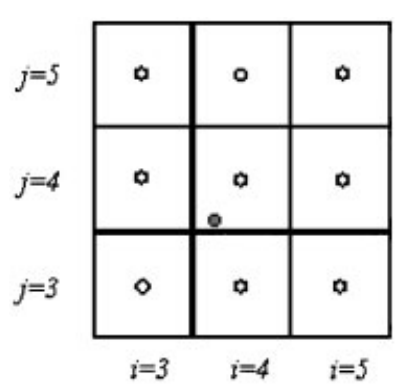

Figure 6. Interpolation square $(6 \times 6)$ for Lagrange interpolation used in the cut-cell approach. 
The Lagrange interpolation polynomial $[30,31]$ is given by (36).

$$
P_{6 \times 6}(x, y)=\sum_{i=0}^{5} \sum_{j=0}^{5}\left(\prod_{k=0, k \neq i}^{5} \frac{x-x_{k}}{x_{i}-x_{k}}\right)\left(\prod_{l=0, l \neq j}^{5} \frac{y-y_{l}}{y_{j}-y_{l}}\right) f\left(x_{i}, y_{j}\right)
$$

where $\left(x_{i}, y_{j}, f\left(x_{i}, y_{j}\right)\right)$ are the pairs of coordinates and values on the open dots (grid points of the numerical solution).

So, this can be performed for every point in the interior. However, for points to be interpolated near the north, east, south, and west boundaries cannot be located in the center of the square. Therefore, the points will be located closer to the edges of the square.

This procedure gives the two-dimensional Lagrange interpolation polynomial, which matches the values at the given points.

2.5.2. Complex boundary. For interpolation near an irregular geometry, in general, 36 surrounding points or conditions have to be found. The system that has to be solved can be cast as Equation (37).

$$
\left[\begin{array}{cccccc}
x_{1}^{5} y_{1}^{5} & x_{1}^{5} y_{1}^{4} & \cdots & x_{1} & y_{1} & 1 \\
x_{1}^{5} y_{1}^{5} & x_{1}^{5} y_{1}^{4} & \cdots & x_{1} & y_{1} & 1 \\
\vdots & \vdots & & \vdots & \vdots & \vdots \\
x_{35}^{5} y_{35}^{5} & x_{35}^{5} y_{35}^{4} & \cdots & x_{35} & y_{35} & 1 \\
x_{36}^{5} y_{36}^{5} & x_{36}^{5} y_{36}^{4} & \cdots & x_{36} & y_{36} & 1
\end{array}\right]\left[\begin{array}{c}
c_{1} \\
c_{2} \\
\vdots \\
c_{35} \\
c_{36}
\end{array}\right]=\left[\begin{array}{c}
f\left(x_{1}, y_{1}\right) \\
f\left(x_{2}, y_{2}\right) \\
\vdots \\
f\left(x_{35}, y_{35}\right) \\
f\left(x_{36}, y_{36}\right)
\end{array}\right]
$$

where $\left(x_{k}, y_{k}, f\left(x_{k}, y_{k}\right)\right)$ are the pairs of coordinates and values on the grid points. Points on the wall and symmetrical opposites of the mass centers are used in the simulation, which can also be used for the interpolation.

This approach can lead to ill-conditioned matrices and large round-off errors, because the coordinates of the points can be very close to each other. In order to avoid this, several adaptations have been made in this approach:

- In order to improve the conditioning of the matrices row scaling has been applied. This leads to a decrease in the condition numbers.

- The coordinates of the 36 conditions used for determining the systems have been shifted to around the origin.

- Choosing the points for interpolation is very important. Because the pressure is interpolated, the pressure part of the wall condition can also be used. Using the wall condition, the mass center and four cell centers per row give a much better conditioning of the matrices.

The final choice of the interpolation points in the vicinity of the wall can be seen in Figure 7. The gray dot is the point to be interpolated. For $i=1, \ldots, 6$, on the open squared dot the wall condition is used, furthermore, the black dot and four cell centers east of the black dot are used, in order to have 36 conditions. 


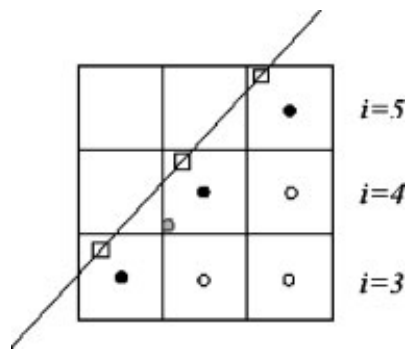

Figure 7. Interpolation area in the vicinity of the wall.

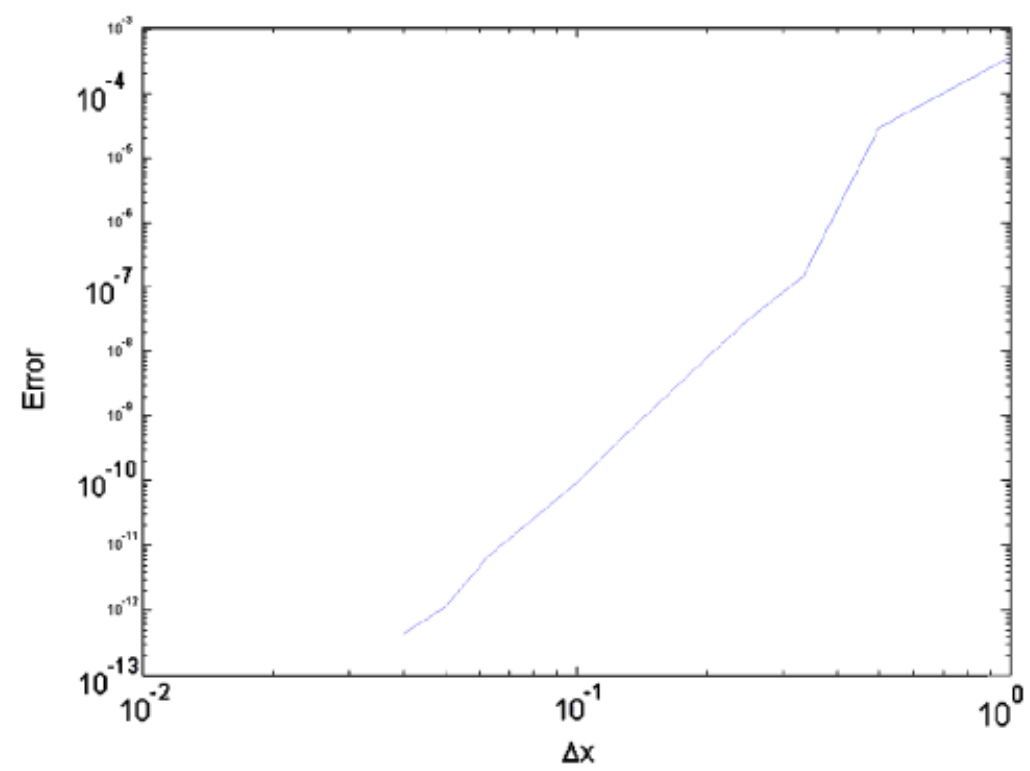

Figure 8. The error versus grid size for the proposed Lagrange interpolation scheme used for interpolation to the common grid.

2.5.3. Testing the interpolation method. The interpolation method has been tested with the analytical function, Equation (38), which satisfies the wall condition $\partial p / \partial n=0$ :

$$
f\left(x^{\prime}\right)=C_{1} \cos \left(x^{\prime}\right)
$$

where $x^{\prime}$ is the orthogonal distance to the wall and $C_{1} \in \Re$.

This function is interpolated from different grids to the common grid. The common grid has grid spacing $\Delta x=0.025$ and $C_{1}=10^{-3}$.

From Figure 8, it appears that the order of accuracy of interpolation method is about 6, which is satisfactory to match the order of accuracy of the numerical scheme for the governing wave equation, which is 4 . 


\section{RESULTS AND DISCUSSION}

Five test cases have been selected to assess the performance of the presented approach: (i) radiation from a baffled piston in an open domain: linear and nonlinear model; (ii) reflection of an acoustic pulse on an oblique wall; (iii) a wave generated by a baffled piston and reflected from an oblique wall: linear model; (iv) wave generated by a piston and reflected by an elliptical solid object; and (v) array of baffled piston.

\subsection{Radiation from a baffled piston}

A uniform circular piston in an infinite rigid baffle is a good starting point for investigating the radiation of sound from a boxed loudspeaker. The physical problem is to find the sound field generated by a vibrating piston surrounded by a rigid baffle. In this paper, we will present the two-dimensional case. This problem is chosen to evaluate the field equation solver in the absence of the rigid wall.

The origin of the coordinate system will be chosen in the center of the piston, as shown in Figure 9. We use the following dimensionless variables with respect to the following scales:

- Length scale $=$ diameter of piston, $2 a$;

- Time scale $=2 a / c$;

- Velocity scale $=$ speed of sound, $c$;

- Density scale $=$ undisturbed density, $\rho_{0}$;

- Pressure scale $=\rho_{0} c^{2}$

- Frequency scale $=c / 2 a$.

3.1.1. Two-dimensional linear baffled piston. The linear Euler equations are as follows:

$$
\begin{array}{r}
\frac{\partial \mathbf{u}}{\partial t}+\nabla p=0 \\
\frac{\partial p}{\partial t}+\nabla \cdot \mathbf{u}=0
\end{array}
$$

The initial conditions are set as follows:

$$
\begin{array}{r}
u_{i}(x, y)=0 \\
u_{j}(x, y)=0 \\
p(x, y)=0
\end{array}
$$

For this problem, two boundary conditions are used, at the wall, and in the far field. The boundary conditions at the wall with the piston are

$$
u_{j}(x, 0, t)= \begin{cases}V_{0} \cos (\omega t), & (x, 0) \in \text { piston } \\ 0, & \text { otherwise }\end{cases}
$$

where $\omega$ is the angular frequency of the piston and $V_{0}$ is the amplitude of the displacement. For low-amplitude signals, the solution can be obtained using the linear equation. Therefore, the value of $V_{0}$ influences only the amplitude of the solution, and none of the other wave parameters. For this reason we can make $V_{0}$ equal to 1 . 


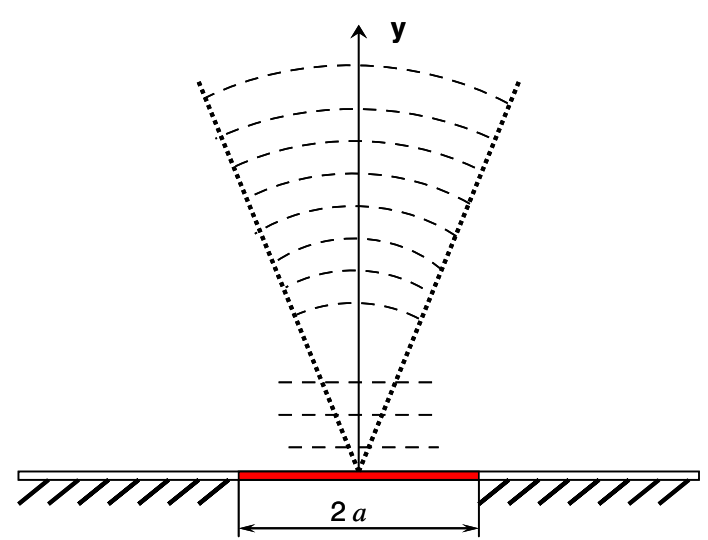

(a)

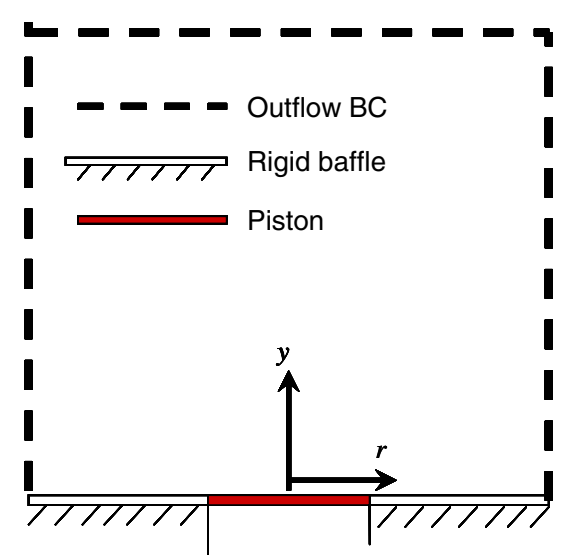

(b)

Figure 9. Radiation from a baffled piston: (a) general description and (b) boundary condition.

The outflow boundary condition is based on the acoustic radiation condition of Tam and Webb [23]:

$$
\begin{array}{r}
\frac{\partial \mathbf{u}}{\partial t}+\nabla p=0 \\
\frac{\partial p}{\partial t}+\frac{\partial p}{\partial x} \cos \theta+\frac{\partial p}{\partial y} \sin \theta+\frac{p}{2 R}=0
\end{array}
$$

where $R$ is the distance from the origin of the $x$-axis (center of the piston) to the point $(x, y)$. The analytical solution is given by the Rayleigh integral [21,22].

The piston behavior is presented in terms of the Helmholtz number, $k a$, where $k$ is wavenumber, and $a$ is the radius of the piston

$$
k a=\frac{\omega a}{c}
$$

Next, we take a closer look at the wave behavior by studying the directivity factor. The directional characteristic of a source is described by the amplitude directivity factor $D$, defined as the pressure at any angle to the pressure on the angle of maximum pressure:

$$
D(\theta)=\frac{p(R, \theta)}{p(R, \pi / 2)}
$$

where the pressure is computed at any arbitrary time $t$. It is clear that the radiation will be strongest on the $y$-axis. This is the reason for which we took maximum pressure at $\theta=\pi / 2$. The directivity factor indicates how effectively the source concentrates its available acoustic power into a preferred direction.

In case that $\mathrm{ka}$ is low, the piston's dimension is small compared with the wavelength of the wave, and in this case the behavior of a piston is like a point source, the directivity pattern does not have a preferred direction. When $k a \gg 1$, the directive function has nulls, and between the nulls are secondary radiation maximum, of monotonically decreasing prominence. The number of nulls 


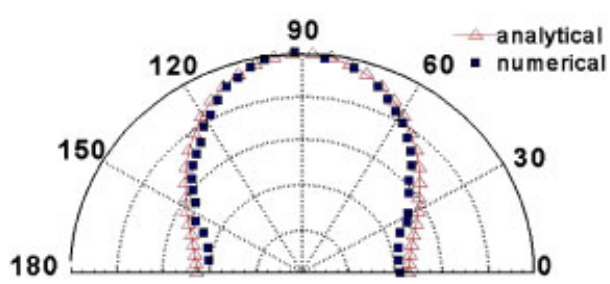

(a)

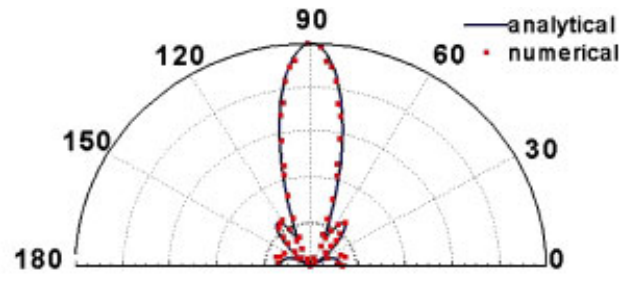

(b)

Figure 10. Radiation from a baffled piston: beam patterns $|D(\theta)|$-two-dimensional linear piston: (a) $k a=2$ and (b) $k a=8$.

and secondary maximum are determined by the size of $k a$, where $a$ is the radius of piston, and $k a=2 \pi a / \lambda$. In other words, the number of lobes increases with the value of $k a$. In our calculation, we compare the analytical and numerical values of beam pattern for two cases, $k a=2$ and 8 . As shown in Figure 10, the numerical solution compares favorably with the analytical beam pattern.

3.1.2. Two-dimensional nonlinear baffled piston. The governing equations are as follows:

$$
\begin{aligned}
& \frac{\partial \rho}{\partial t}+\frac{\partial\left(\rho u_{j}\right)}{\partial x_{j}}=0 \\
& \frac{\partial\left(\rho u_{i}\right)}{\partial t}+\frac{\partial\left(\rho u_{i} u_{j}\right)}{\partial x_{j}}+\frac{\partial p}{\partial x_{i}}=\frac{\partial \tau_{i j}}{\partial x_{j}} \\
& \frac{\partial p}{\partial t}+u_{j} \frac{\partial p}{\partial x_{j}}+\gamma p \frac{\partial u_{j}}{\partial x_{j}}=0
\end{aligned}
$$

where

$$
\tau_{i j}=\frac{1}{R_{D}}\left(\frac{\partial u_{i}}{\partial x_{j}}+\frac{\partial u_{j}}{\partial x_{i}}\right)
$$

where $R_{D}=2 a c / v$ is the Reynolds number based on the diameter of the piston, $v$ is the kinematic viscosity of the fluid, and $\gamma$ is the ratio of specific heat.

The initial conditions are as follows:

$$
\begin{aligned}
& \rho=1 \\
& p=1 / \gamma \\
& u=0 \\
& v=0
\end{aligned}
$$

The boundary conditions at the wall with the piston are given in Equation (41).

For outflow boundary, we use PML, given by (16) that is finished with the outflow boundary condition designed by Tam and Webb [23] (Equation (18)). 
No exact solutions exist in this case. To help assess the performance of our approach we will evaluate the solution of the small amplitude case, which can be approximated by the linear model. We will present the results for two scenarios: low frequency $(k a=2)$, and high frequency $(k a=8)$.

3.1.2.1. Low frequency $(k a=2)$. The computation is done in a domain $(x, y) \in[-5,5] \times[0,10]$ and a grid $161 \times 161$. The time step is based on $\mathrm{CFL}=0.2$, where

$$
\mathrm{CFL}=c \frac{\Delta t}{\min (\Delta x, \Delta y)}
$$

First, we present a solution for which $V_{0}$ is small. In this case, the linear model can approximate the solution. The solution and contour plot for $V_{0}=0.01 \mathrm{c}$ are presented in Figure 11(a) and (b). We can see that the solution is very close to the linear approach. This is expected because we have the case of a small signal, when the solution can be approximated very well with the linear behavior.

Blackstock [22] shows that in the plane of progressive waves, the speed of sound can be approximated by $c+\beta u$, where $\beta$ is the coefficient of nonlinearity. Not all points on the wave move at the same speed. At the point with $u=0$, the wave essentially moves at the speed of sound $c$. However, at the peak, where $u$ has its greatest value due to nonlinear effects, the wave travels the fastest. At the trough, where $u$ is the lowest, the wave is slowest. Consequently, the peak tends to catch up with the trough, as shown in sketches (a), (b), and (c) in Figure 12. The multi-valued waveform is physically impossible. The effects of viscosity and heat conduction prevent actual multi-values. Dissipation is important whenever any segment of the waveform becomes very steep, or when shocks are formed. The computational results show that when $V_{0}$ increases the solution becomes steeper and the dissipation becomes very important because the speed of the sound is bigger $(c+\beta u)$. This is illustrated in Figure 13(a)-(c), where the linear solution is plotted to emphasize that dissipation increases as the initial amplitude grows. In Figure 13(d), we present
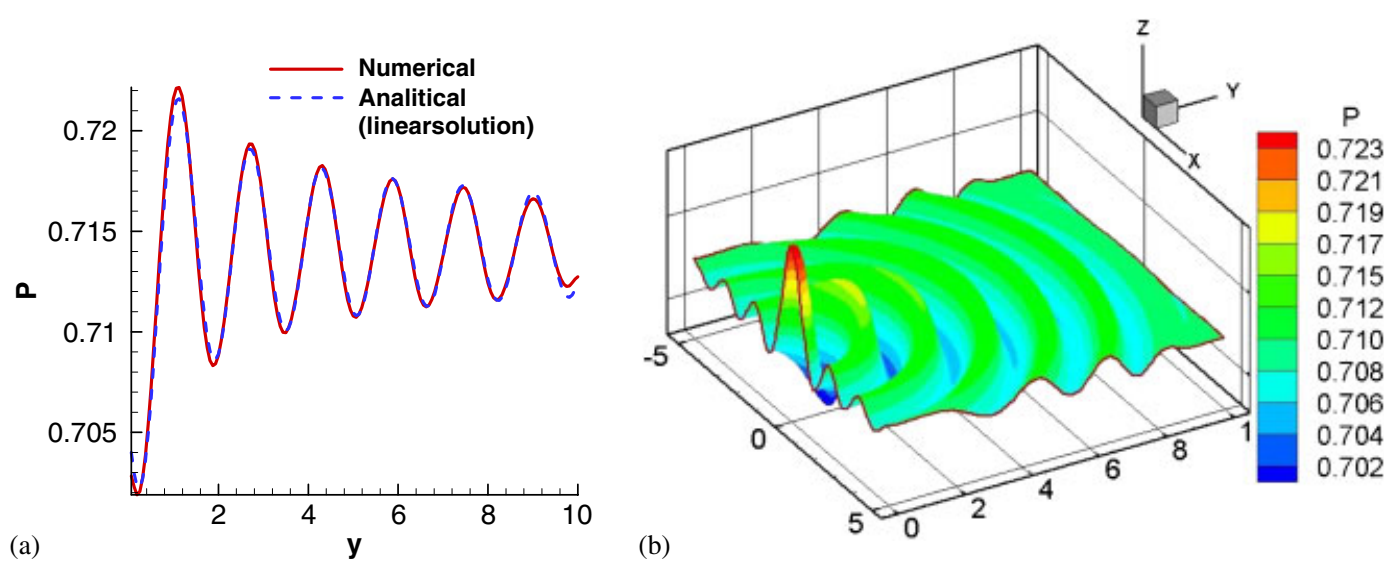

Figure 11. Nonlinear baffled piston: $t=12, \mathrm{CFL}=0.2, V_{0}=0.01 c_{0} ; k a=2$ : (a) axial solution and (b) contour plot solution. 


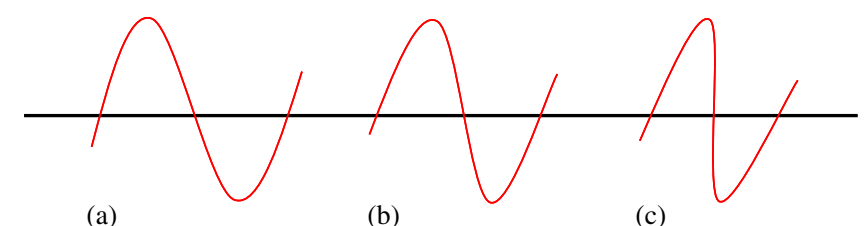

(a)

(b) (c)

Figure 12. Cumulative distortion of a plane progressive wave.
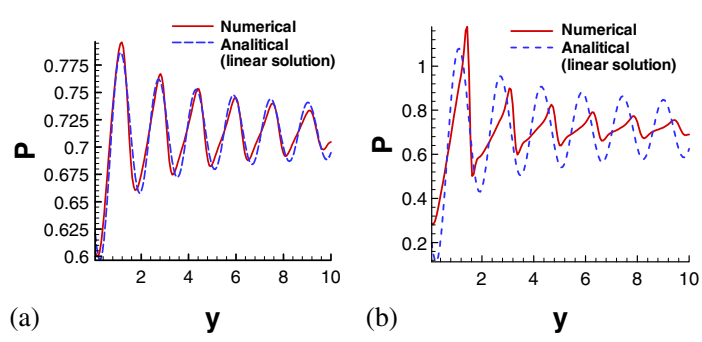

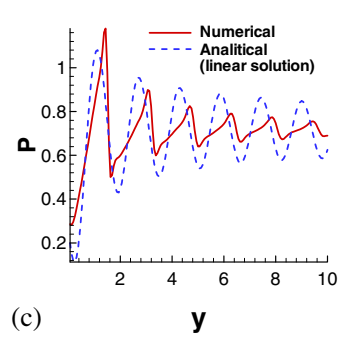

y

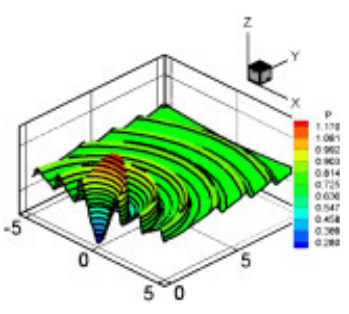

(d)

Figure 13. Axial solution: $t=12 ; k a=2, \mathrm{CFL}=0.2-$ Nonlinear baffled piston: (a) $V_{0}=0.1 c_{0}$; (b) $V_{0}=0.3 c_{0}$; (c) $V_{0}=0.5 c_{0}$; and (d) three-dimensional contour plot, $V_{0}=0.5 c_{0}$.

the contour plot of a dissipative solution. We can see that the boundary condition assures again a negligible reflection from the boundary.

3.1.2.2. High frequency $(k a=8)$. The computation is performed in a domain $(x, y) \in[-2.5,2.5] \times$ $[0,5]$ and on a grid system of $251 \times 251$ nodes. The time step is based on CFL $=0.2$. In this case, we use a finer grid to be able to capture the behavior of high frequency. As shown in Figure 14 , for the case of weak signals $\left(V_{0}=0.01\right)$, the solution is well approximated with a linear behavior, except that the nonlinear computation captures the detailed behavior around the piston, namely, the diffraction because of the discontinuity around the edge of the piston (see Figure 14(b)). In the case of a high frequency, wave radiated from a baffled piston dissipates faster than in the case of low frequency. This happens because the speed of a high-frequency wave is larger, hence the speed of the sound $(c+\beta u)$ is higher, and the diffusion becomes more powerful. This phenomenon is observed in our computation (see Figure 15(a)). Again, we notice that the actual boundary condition yields a negligible reflection also in this case (see Figure 15(b)).

\subsection{Array of baffled pistons}

The problem consists of solving the radiation interference from more than one piston. This is shown in Figure 16, where three pistons are considered. The challenge of the numerical solution will consist of capturing the behavior of the new waves, particularly the range of wavenumbers. Again the exact solution cannot be obtained; hence, we cannot make a quantitative evaluation of the results and the qualitative evaluation will demonstrate the correctitude of our results. The proposed approach is solved using the Navier-Stokes equations, where the wall boundary conditions and piston source characteristics for wall-embedded pistons, Equations (15)-(16), and the outflow 


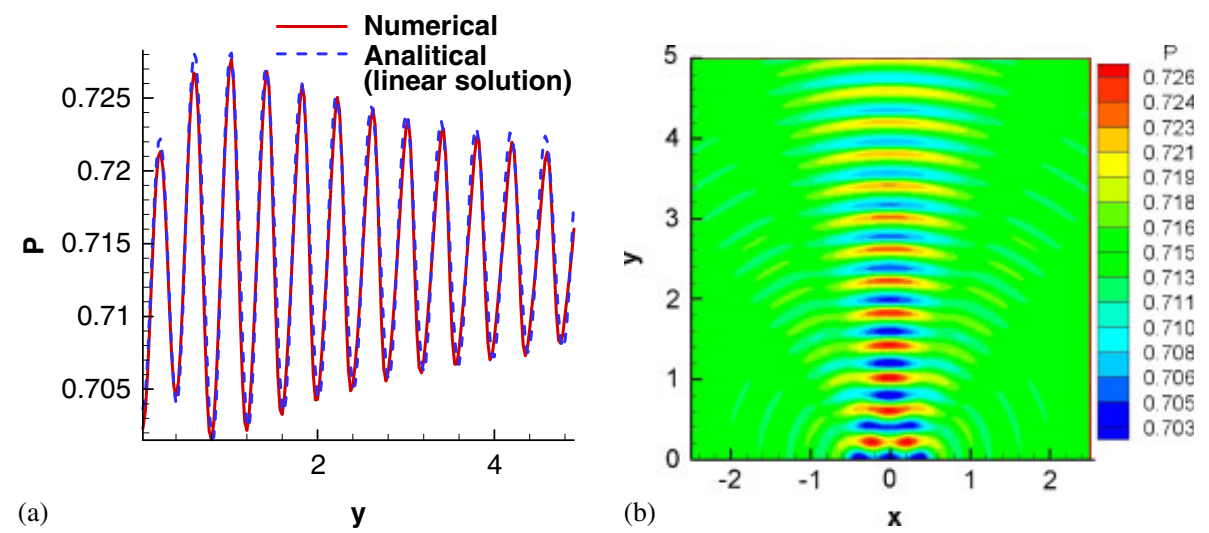

Figure 14. Solutions of nonlinear baffled piston: $t=12, \mathrm{CFL}=0.2: V_{0}=0.01 c_{0} ; k a=8$ : (a) pressure profiles along the axial direction and (b) pressure contour plot.
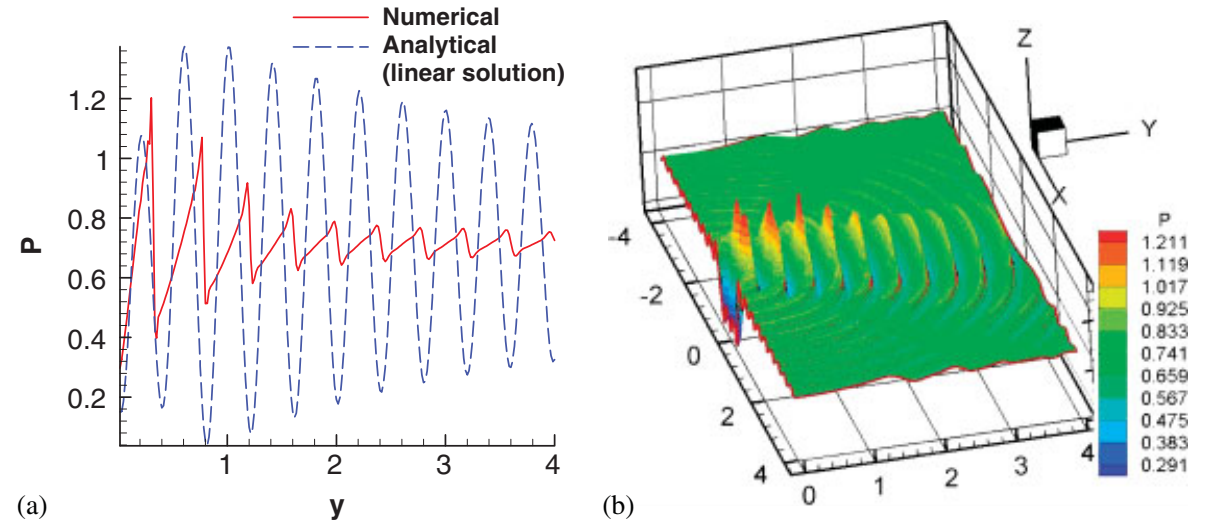

Figure 15. Radiation wave from a baffled piston: $t=12, V_{0}=0.5 c_{0} ; k a=8$; nonlinear baffled piston: (a) pressure profiles along the axial direction and (b) pressure contour plot.

boundary conditions are handled using the PML and acoustic radiation conditions of Tam and Webb [23], Equations (16) and (18).

Next, we study the interference of the waves of three sources when the source is characterized by low frequency $(k a=2)$ and high frequency $(k a=8)$.

3.2.1. Low frequency $(k a=2)$. In case of the low frequency, the source is characterized by the directivity pattern without nulls and maxims; in other words, the radiated waves do not have a preferred direction. With weak signals (e.g. $\left.V_{0}=0.01\right)$ the interference propagates largely along the radial directions from the sources (see Figure 17(a) and (b)). As signals strengthen, the waves dissipate quickly, and the maxima and minima follow the directivity pattern of the sources (see Figure 17(c) and (d)). 


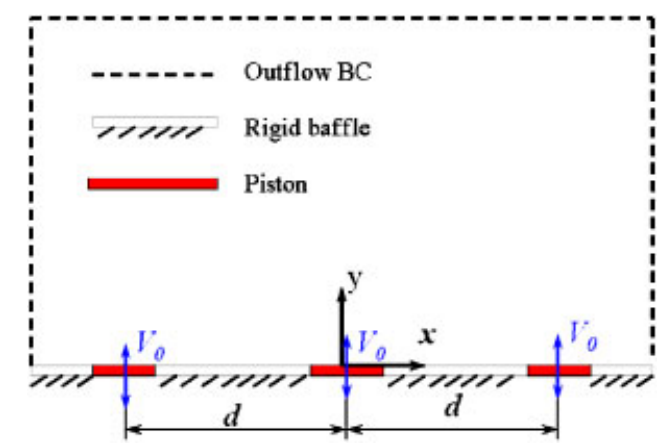

Figure 16. Array of the baffled pistons: general presentation.
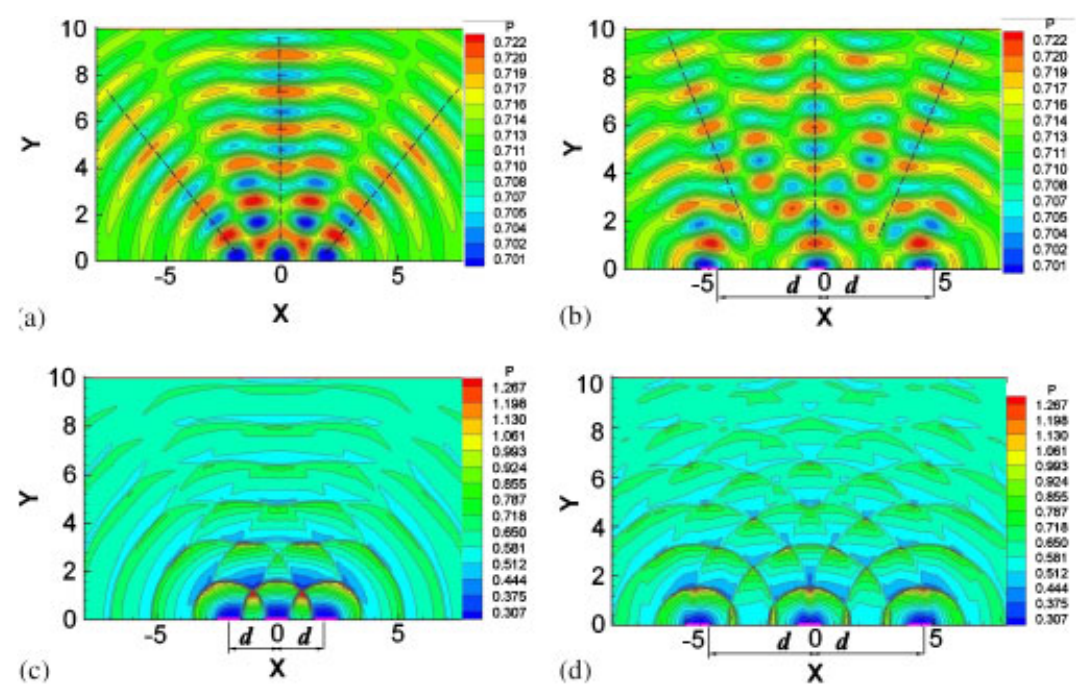

Figure 17. Radiated wave from array of the three baffled pistons: $t=12 ; k a=2$ : (a) $V_{0}=0.01$, $d=2$; (b) $V_{0}=0.01, d=4.5$; (c) $V_{0}=0.5, d=2$; and (d) $V_{0}=0.5, d=4.5$.

3.2.2. High frequency $(k a=8)$. A high-frequency source is characterized by an efficiently radiated wave in the direction of the axis of the piston. In this case, the interference between radiated waves becomes more pronounced as the distance between pistons decreases. When the pistons are close enough, the interference waves are very well defined, and their position and power depend on the position of the source. This behavior is captured very well by our computation as can be seen in Figure 18. In the case that the piston emits a strong signal, the shock wave appears very close to the piston and waves dissipated rapidly (see Figure 19).

\subsection{Reflection of a pulse on an oblique wall}

A simple example appropriate for checking the performance of the cut-cell approach is the reflected wave from an oblique wall, illustrated in Figure 20. Even though this problem can be solved by 

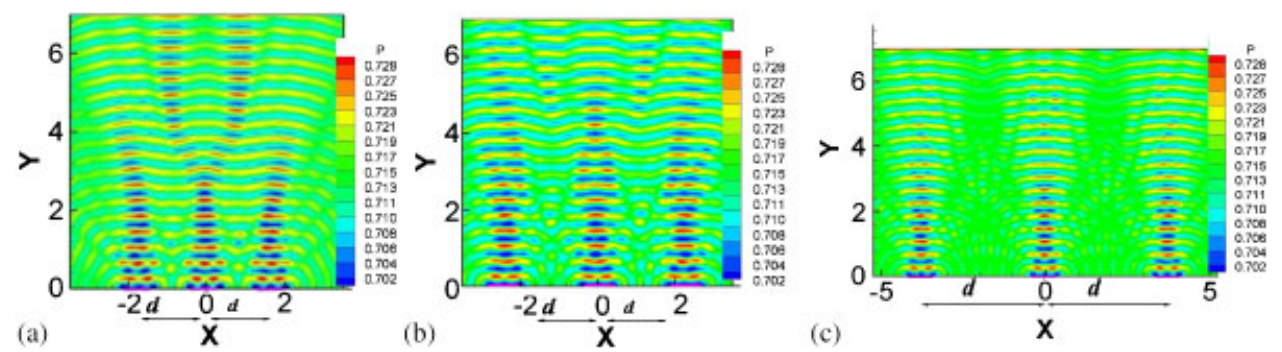

Figure 18. Radiated wave from array of the three baffled pistons: $t=12 ; V_{0}=0.01 ; k a=8$; distance between the center of the piston (d): (a) 1.75; (b) 2.3; and (c) 3.75 .

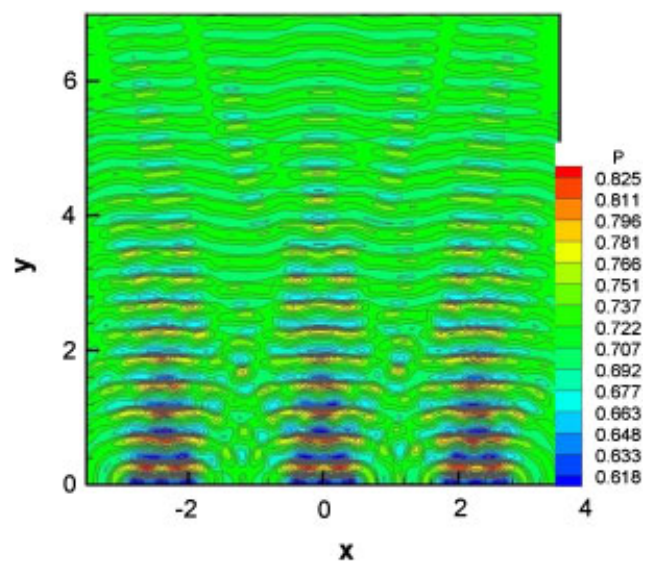

Figure 19. Radiated wave from array of the three baffled pistons: $t=12 ; V_{0}=0.1 ; \omega=16 ; d=2.3$.

placing the wall parallel to the grid line, we purposely arrange the wall so that it is an oblique angle to the grid line. This offers a direct evaluation of the cut-cell technique. In this example, the sound hits the wall and reflects. The linearized Euler Equation (39) characterizes the problem. The initial condition is

$$
p(x, y)=\exp \left\{-\ln (2)\left[\left(\frac{x-x_{0}}{b}\right)^{2}+\left(\frac{y-y_{0}}{b}\right)^{2}\right]\right\}
$$

where $b=\frac{1}{6}$. The values $x_{0}$ and $y_{0}$ are chosen so that the distance from the point $\left(x_{0}, y_{0}\right)$ to the oblique solid wall is equal to 1.5 .

The outflow boundary condition is the same as that used in the previous linear test problem. The solid wall boundary conditions for pressure and velocity are given in Equation (31). The computation is performed on a Cartesian grid that is characterized by $\Delta x=\Delta y=0.05$ and $\mathrm{CFL}=0.5$.

Figure 21 illustrates the solid boundary intersecting a Cartesian mesh. The boundary cell can remain independent, like in $A B C K$, or it can merge with a neighboring cell. For example, $C I D$ merges with $K C I J$. The computation of a dependent variable, such as pressure, in the cut-cell 


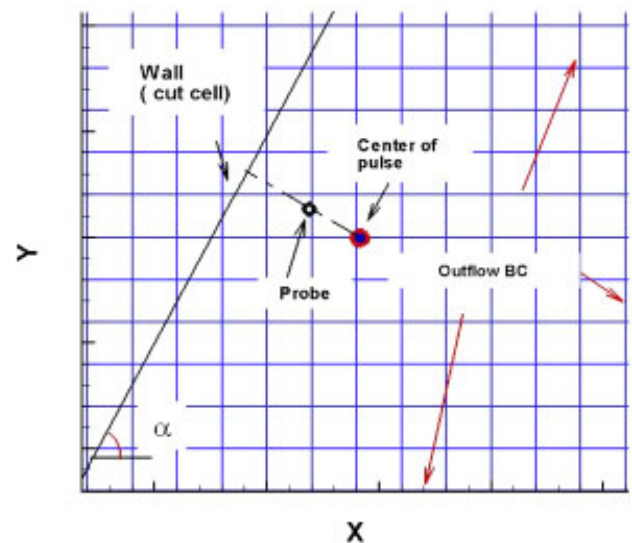

Figure 20. Schematic illustration of reflection of the pulse on an oblique wall.

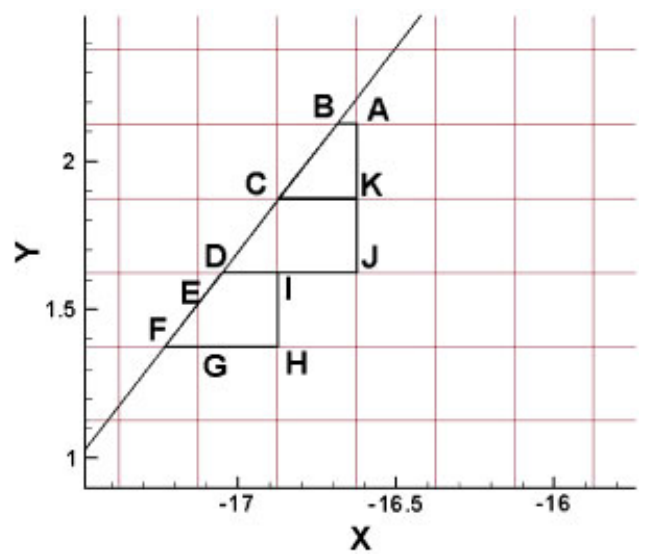

Figure 21. Reflection of the pulse on an oblique wall: cell around boundary.

EFGH I can be written from wave equation (39) in the form:

$$
\frac{\partial \bar{p}}{\partial t}+\frac{1}{s_{E F G H I}}\left(u_{C D}^{x} d y_{C D}+u_{J K}^{x} d y_{J K}-u_{I D}^{y} d x_{I D}-u_{F G}^{y} d x_{F G}-u_{G H}^{y} d x_{G H}\right)=0
$$

where the values of $u^{x}$ and $u^{y}$ represent the values of the functions $u_{i}$ and $u_{j}$ in the middle of the segment. The value of the parameter is approximated using: (i) the OPC scheme on faces $H I$ and $G H$; (ii) fourth-order polynomial on faces $D I$ and $F G$; and (iii) Equation (31) on face $D F$ (boundary).

To evaluate the performance of the cut-cell approach, Figure 22 shows the pressure history from time $=0-4$ and for three wall angles: $90^{\circ}, 81^{\circ}$, and $63^{\circ}$. As we see from the figure, there is a good agreement between these solutions. Figure 23 shows the pressure contours at three time instants by placing the solid wall at a $63^{\circ}$ inclination. 


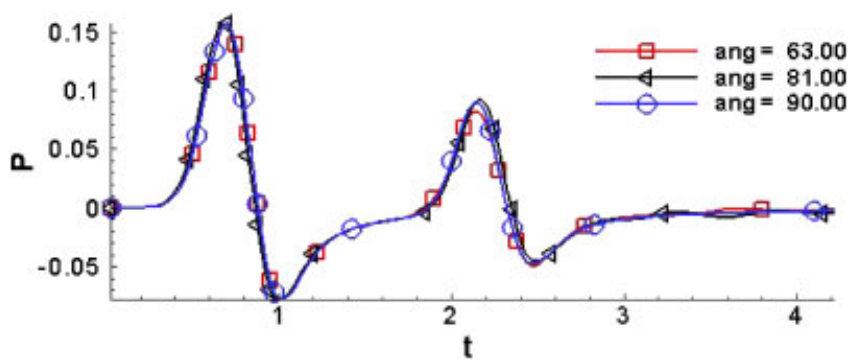

Figure 22. Reflection of the pulse on an oblique wall: history of pressure for different angles of wall.
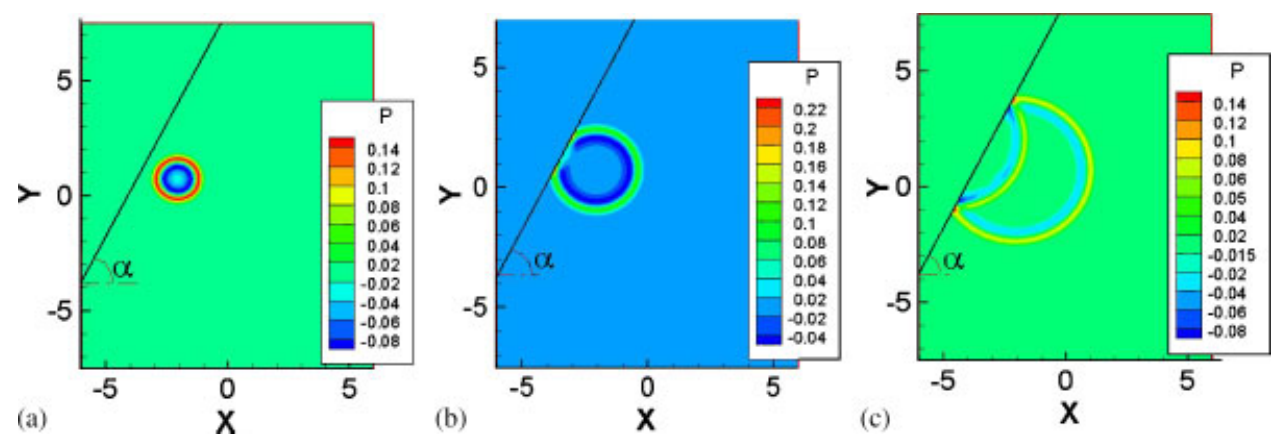

Figure 23. Reflection of the pulse on an oblique wall, $\alpha=63^{\circ}$ : (a) $t=0.8$; (b) $t=1$.6; and (c) $t=3.2$.

\subsection{Wave generated by a baffled piston and reflected on an oblique wall}

This problem is based on the combined characteristics of the previous two cases, and is an example where the exact solution does not exist and the performance of the numerical techniques is assessed based on grid refinement. The wave generated by a piston is reflected on an oblique wall. The linearized Euler equations (Equation (39)) are used in this test case, and the initial condition is the same as that of the baffled piston, Equation (40).

The rectangular domain over which we do the computation is similar to the previous case (see Figure 24) with: $(x, y) \in[-6,6] \times[0,15]$. The bottom of the domain is a piston mounted on a plane rigid baffle; hence the boundary conditions are given by Equation (41). The velocity and pressure on the solid and open boundaries are identical to those used in the previous case.

The piston presents the same characteristics as the piston from the previous case: $V_{0}=1$ and $k a=2$. The solution is obtained using the uniform grid $\Delta x=\Delta y=0.05$, and with a time step of $\mathrm{CFL}=0.5$. Figure 25 highlights the pressure contours at different time instants, especially with regard to the pattern of the wave generated by the baffled piston, and influence of the wall.

\subsection{Wave from a baffled piston around an elliptical solid object}

The purpose of this case is to test the ability of cut-cell method. The computational domain (sound field) is a square with domain $(x, y) \in[-5,5] \times[0,10]$ and a baffled piston with radius 0.5 is mounted at the bottom side of the domain. In this problem, the low-amplitude signal condition is 


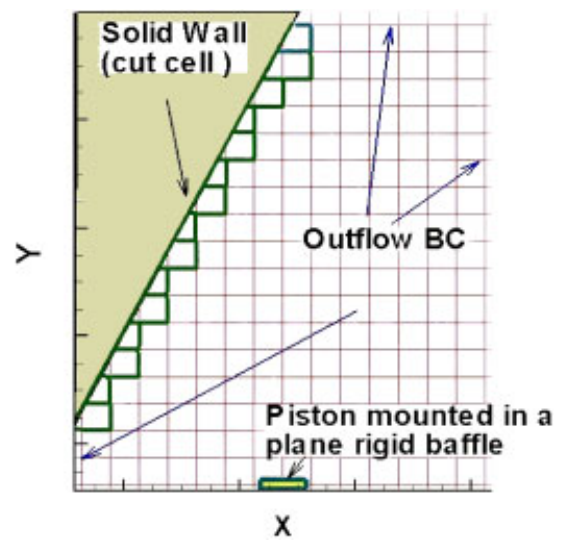

Figure 24. Wave generated by a baffled piston and reflects on an oblique wall: general description of the domain.
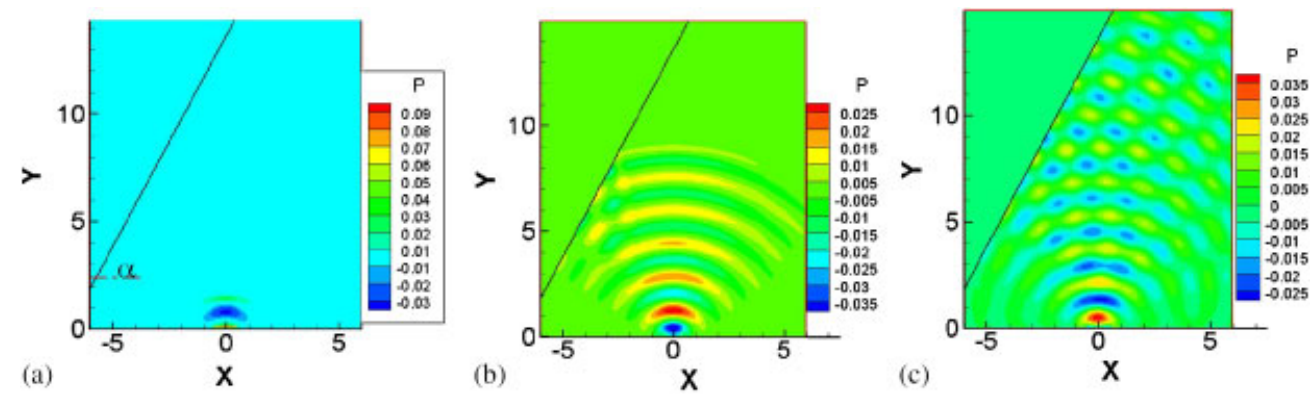

Figure 25. Wave generated by a baffled piston and reflects on an oblique wall, $\alpha=63^{\circ}$ :

(a) $t=1.5$; (b) $t=9.4$; and (c) $t=25.0$.

assumed and the linear Euler equations are used. The CFL number is 0.5 in this case. Specifically, a small solid ellipse is placed at the center of the domain for which the long axis is 0.5 and the short axis is 0.25 . The center of the ellipse is at $(0 ., 0$.$) , and the ellipse is inclined at an angle of$ $30^{\circ}$. The exact solution does not exist and the performance of the numerical techniques is assessed based on grid refinement.

A grid refinement test is used to assess the convergence of the solution, which Figure 26 shows the geometry and the pressure distribution at $x=0$ along the $y$-axis at $t=10$ based on different grid resolutions. As the grid is refined, the solutions converge toward each other, demonstrating that the cut-cell approach works satisfactorily in regard to yielding unique solutions.

\subsection{Order of accuracy assessment via Richardson extrapolation}

We study the accuracy of the solution for the wave generated by a baffled piston and reflected by an oblique wall case. First, we determine the order of accuracy before the waves, generated by the baffled piston, hit the wall. The solutions $P(\Delta x), P(\Delta x / 2)$, and $P(\Delta x / 4)$ are interpolated to the common grid with grid spacing $\Delta x / 4$, where $\Delta x=0.1$ and the $\mathrm{CFL}=0.5$, which determines 


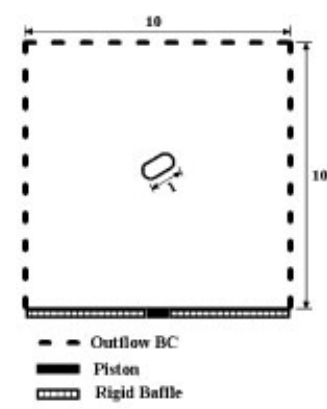

(a)

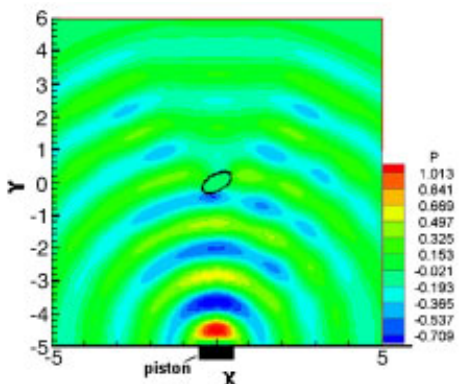

(b)

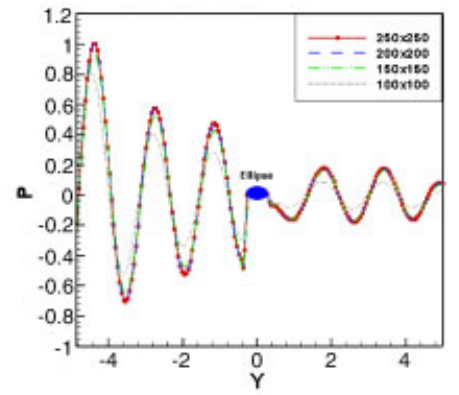

(c)

Figure 26. Geometry and pressure distribution of different grid sizes of linear baffled piston with an oblique ellipse: $\omega=4, V_{0}=1.0, t=10$ : (a) geometry; (b) pressure contour; and (c) axial solution.
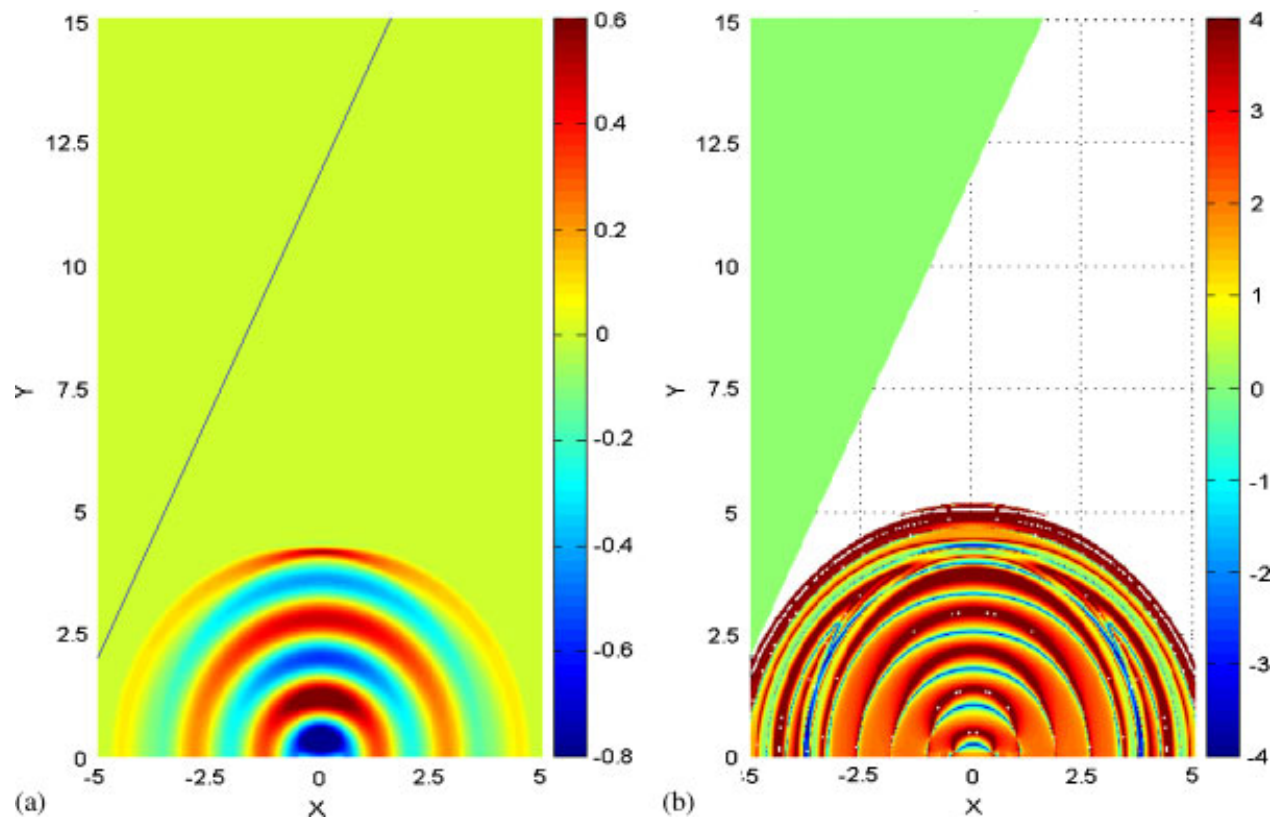

Figure 27. (a) Numerical solution of wave originated from a baffled piston and reflected by an oblique wall case with grid spacing $\Delta x / 4$ and CFL-number of 0.5 at $t=4.2$ and (b) order of accuracy.

the time step. The order of accuracy will not be determined if $P(\Delta x / 4)=P(\Delta x / 2)$ within round-off error. From Figure 27, it appears that the order of accuracy is space dependent. The derived order of accuracy is 4 , but the order of accuracy in practice is much less. This is caused by the presence of discontinuities in space and time between piston and wall. Shyy et al. [30] have considered Navier-Stokes computations and demonstrated that for flows with large jumps in velocity profiles, Richardson extrapolation is challenged. The various aspects of Richardson extrapolation in the context of wave propagations will be discussed next. 
3.6.1. Impact of discontinuities. The piston has length 1 and piston $\in\left[-\frac{1}{2}, \frac{1}{2}\right]$. Therefore, the boundary condition in Equation (41) at $y=0$ has two discontinuities at $x=-\frac{1}{2}$ and $\frac{1}{2}$. Furthermore, the initial condition on the piston is $V_{0}$, while the rest of the domain has initial condition 0 . This gives another discontinuity on the piston in the initial condition.

These discontinuities can be removed by replacing the boundary condition at $y=0$ by

$$
u_{j}(x, 0, t)=V_{0} \sin (\omega t)\left[\frac{1}{2} \cos (2 \pi x)+\frac{1}{2}\right]
$$

where the time-dependent sine term makes the initial condition continuous and the cosine term makes the boundary condition at $x=-\frac{1}{2}$ and $\frac{1}{2}$ continuous.

The result of replacing the discontinuous boundary condition on the south boundary by a continuous one is given in Figure 28. From this figure it appears that the order of accuracy has

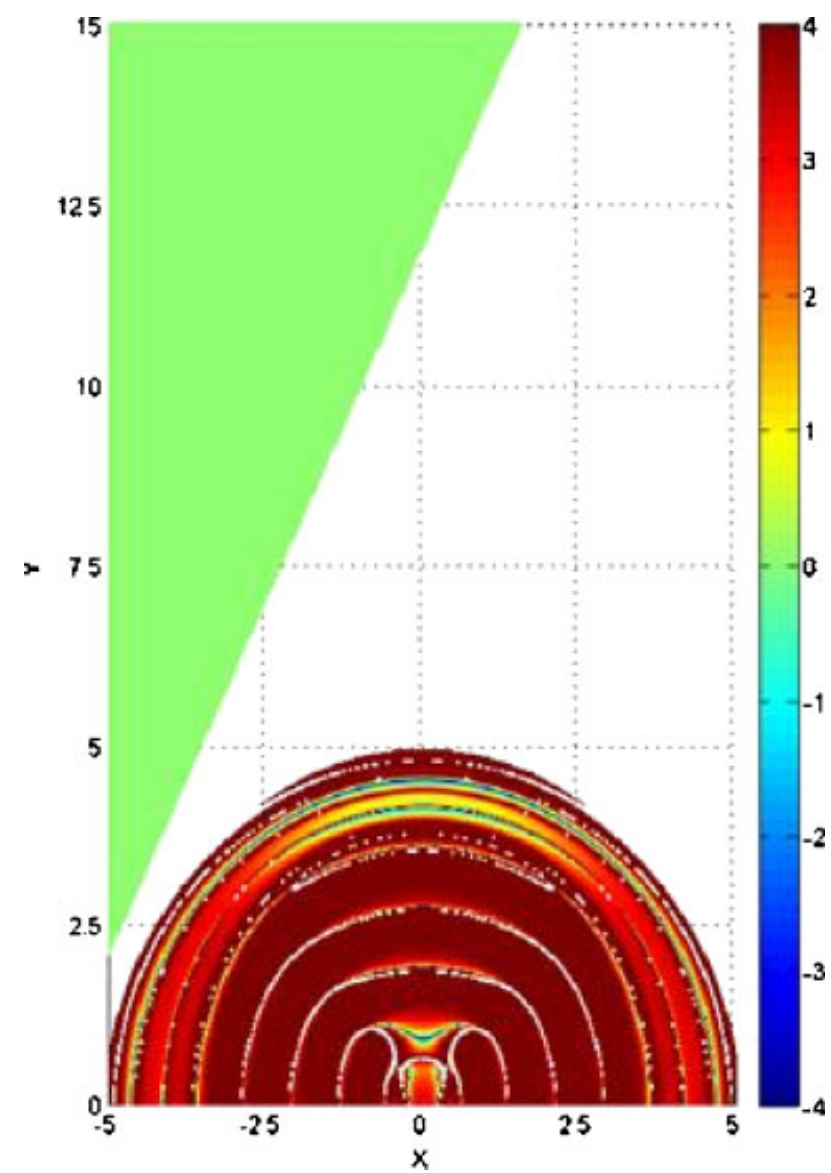

Figure 28. Order of accuracy of a wave originated from a baffled piston and reflected by an oblique wall case at $t=4.2$. 

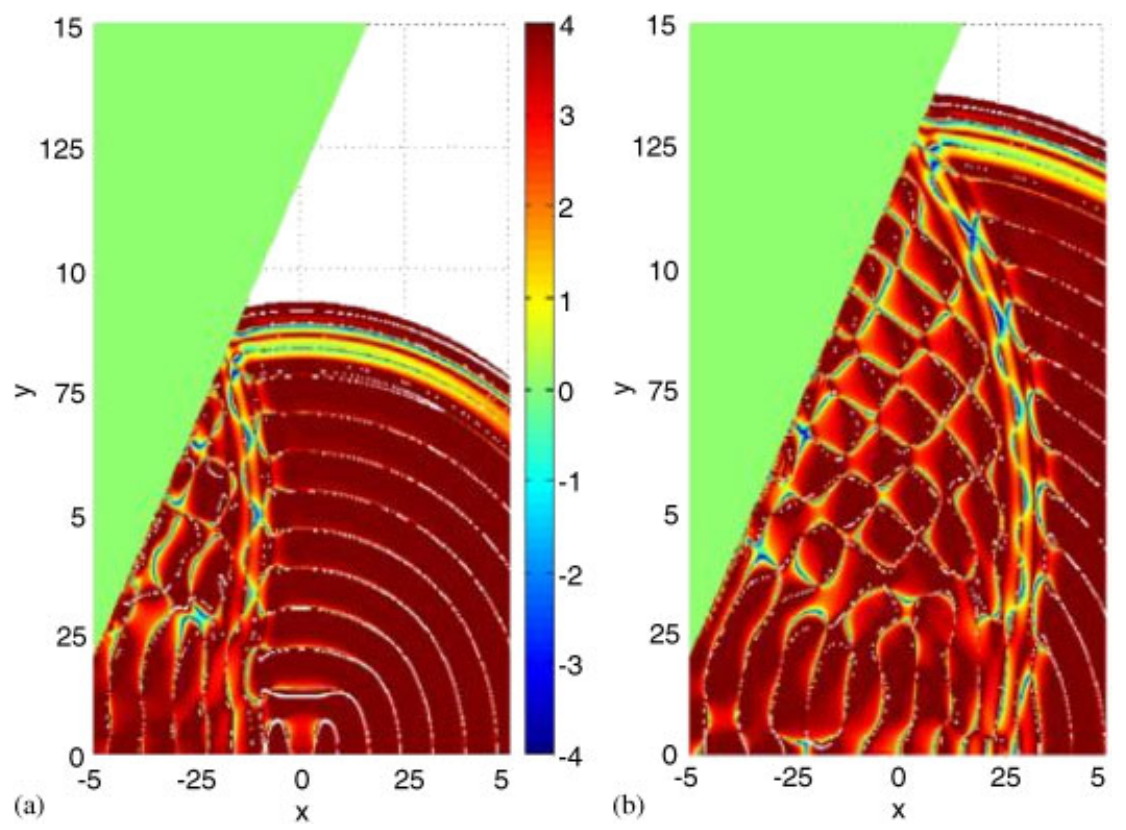

Figure 29. Order of accuracy of a wave originated from a baffled piston and reflected by an oblique wall case: (a) $t=8.4$ and (b) $t=12.6$-west boundary is an oblique wall.

improved. The magnitude of the order of accuracy is about 4, which is the derived order of accuracy.

The white lines correspond with singularities in the order of accuracy. This is caused by the phenomenon that the order of accuracy cannot be determined if $P(\Delta x / 4)=P(\Delta x / 2)$ within round-off error. This phenomenon is conform with Shyy and Garbey [31].

With this approach, substitution of $\Delta t$ in Equation (32), it has been checked that the order of accuracy is CFL-number independent.

3.6.2. Impact of cut-cell method. In order to investigate the impact of the cut-cell method on the order of accuracy, the impact of the outflow boundary condition should be eliminated. This can be carried out implementing an oblique wall on the west boundary, with wall boundary conditions (31). From Figure 29 it appears that the order of accuracy in the reflected wave decreases to about 3 , less in some areas. Thus, it can be concluded that there is a slight negative impact on the order of accuracy.

3.6.3. Richardson extrapolation for accuracy improvement. In order to investigate whether Richardson extrapolation can be an effective tool to improve the solution, two test cases will be investigated. Richardson extrapolation is designed such that it cancels the leading truncation error term in Equation (33). However, there is an additional requirement for wave problems. The extrapolated solution should give a smooth wave again. 


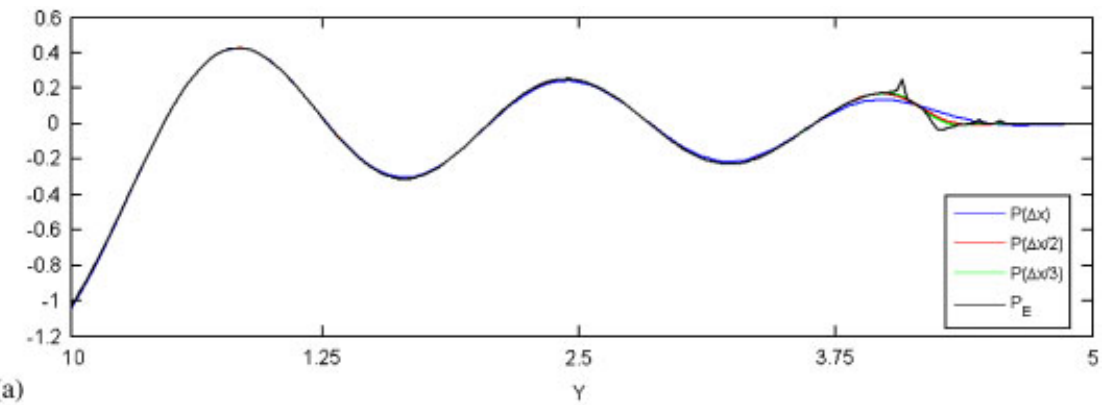

(a)

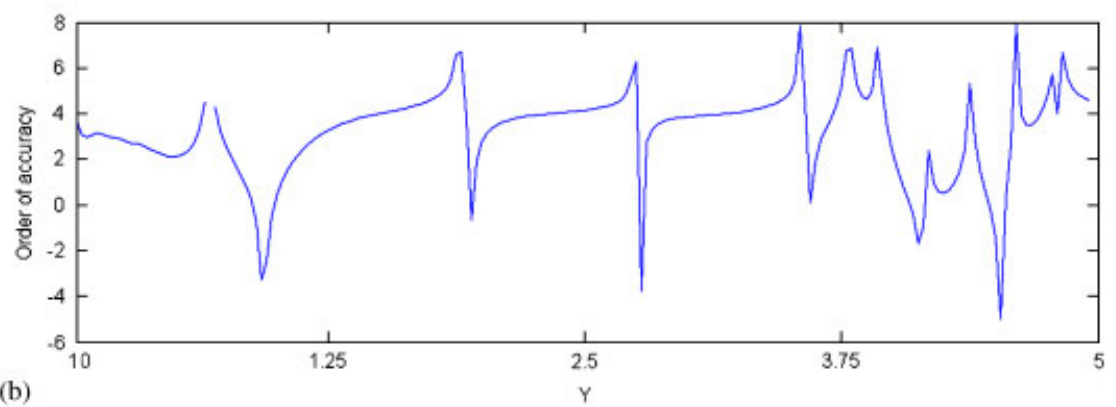

Figure 30. Solution of a wave originated from a baffled piston and reflected by an oblique wall case, along $x=0$ at $t=4.2$ : (a) numerical solutions and extrapolated solution PE and (b) order of accuracy.

3.6.3.1. Baffled piston. Richardson extrapolation is applied to the problem before the waves hit the wall. No extrapolation is needed where singularities appear, because then the solutions are equal within round-off error. Extrapolation is only applied to points on the grid where the solutions are converging, so where the order of accuracy is positive.

From Figure 30 it appears that the extrapolated solution does not satisfy the additional condition of being a smooth wave. In the wavefront, the order of accuracy is very poor and unstructured, which is caused by the discontinuity in the time derivative of the initial condition (50). Consistent with the point made by Shyy et al. [30], this causes unsatisfactory results with Richardson extrapolation.

3.6.3.2. Acoustic pulse. The second test case for investigating Richardson extrapolation is the propagation of an acoustic pulse. The initial condition is given by Equation (48).

Richardson extrapolation has been applied with grid solutions $P(\Delta x / 2), P(\Delta x / 4)$, and $P(\Delta x / 8)$. From Figure 31, it appears that the extrapolated solution is smooth. By the construction of Richardson extrapolation this is an improved solution. However, it cannot be determined how many orders it has improved. The only thing known is that the leading truncation error term is cancelled.

From the two test cases it can be concluded that Richardson extrapolation can be an effective tool to improve the solution if the problem is smooth and does not contain large gradients or discontinuities. 

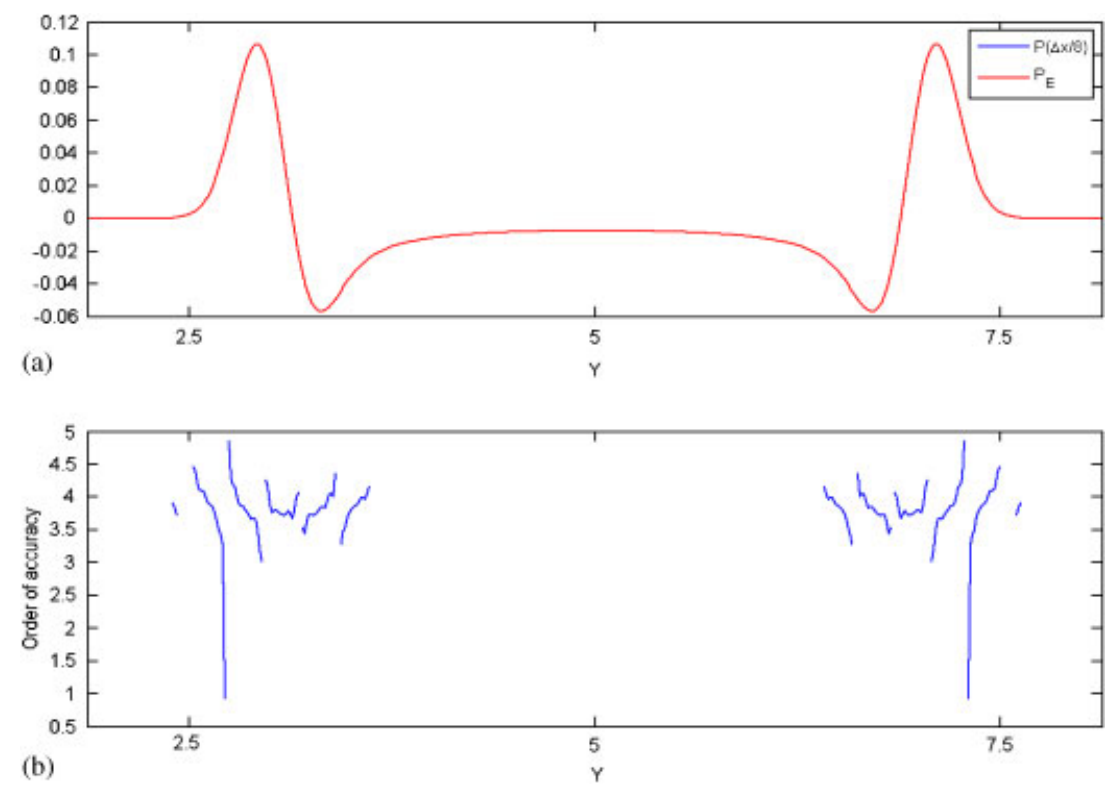

Figure 31. Slices $x=0$ at $t=2$ : (a) finest grid solution and extrapolated solution and (b) order of accuracy.

\section{SUMMARY AND CONCLUSION}

A method based on a high order, finite-volume scheme aimed at optimizing the dispersion and dissipation properties, and the cut-cell technique aimed at handling geometric variations, is presented. The approach is motivated by the need for handling acoustic problems with nonlinearities (using finite volume technique) and complex geometries (using the cut-cell technique). The finite volume-based OPC scheme and the Cartesian cut-cell approach are combined to offer the nominal fourth-order accuracy and geometric flexibility. The computational overhead of the cut-cell approach is modest because the following information needs to be computed only once, unless, of course, if the geometry is time dependent:

- data communication between cells affected by the boundary treatment;

- calculation of area and other geometric information;

- interpolation procedures required for the flux computation in the boundary region.

Numerical computations of the baffled piston have been conducted with both linear and nonlinear models. For nonlinear waves, depending on the flow parameters, the propagation, interference, sharpening, and dissipation of the wave characteristics are well captured. For example, for the weak signal case, since the dissipation is less important, we observe the pattern of interfering waves. For a stronger signal, the shock wave appears close to the pistons, and experiences dissipation quickly. Richardson extrapolation is used to evaluate the performance of the solution technique. It is shown that discontinuities in space and time significantly influence the general order of accuracy of the solution. The cut-cell method has a modest effect on the order of accuracy. It is also demonstrated 
that Richardson extrapolation can be successful in improving the solution only for problems that do not contain high gradients or discontinuities.

Based on the evaluation of the test cases investigated, we conclude that the present approach can be effective in treating aeroacoustics problems with irregular geometry.

\section{ACKNOWLEDGEMENTS}

The present work was supported in part by the NASA Constellation University Institutes Program (CUIP), Ms Claudia Meyer program monitor.

\section{REFERENCES}

1. Hardin J, Hussaini MY. Computational Aeroacoustics. Springer, New York/Berlin, 1992.

2. Tam CKW, Webb JC, Dong Z. Dispersion-relation-preserving finite difference schemes for computational acoustic. Journal of Computational Physics 1993; 107(2):262-281.

3. Hixon R. Evaluation of high-accuracy MacCormack-type scheme using benchmark problems. NASA Contractor Report 202324, ICOMP-97-03, 1997.

4. Kim C, Roe PL, Thomas JP. Accurate schemes for advection and aeroacoustic. Technical Paper 97-2091, AIAA Press, Washington, DC, 1997.

5. Lin SY, Chin YS. Comparation of higher resolution Euler schemes for aeroacoustics computation. AIAA Journal 1995; 33:237-245.

6. Shyy W. A study of finite difference approximations to steady-state, convection-dominated flow problems. Journal of Computational Physics 1985; 57:415-438.

7. Shyy W. Computational Modeling for Fluid Flow and Interfacial Transport. Elsevier: Amsterdam, The Netherlands, 1994, revised printing 1997.

8. Hu FQ, Hussaini MY, Manthey JL. Low dissipation and dispersion Runge-Kutta for computational acoustics. Journal of Computational Physics 1996; 124:177-191.

9. Stanescu D, Habashi WG. 2N-storage low dissipation and dispersion Runge-Kutta for computational acoustics. Journal of Computational Physics 1998; 143:674-681.

10. Nance DV, Viswanathan K, Sankar LN. Low-dispersion finite volume scheme for aeroacoustic application. AIAA Journal 1997; 35(2):255-262.

11. Wang G, Sankar LN. Prediction of rotorcraft noise with a low-dispersion finite volume scheme. AIAA-99-0480, 1999.

12. Cheong C, Lee S. Grid-optimized dispersion-relation-preserving schemes on general geometries for computational aeroacoustic. Journal of Computational Physics 2001; 174:248-276.

13. Wang ZJ, Chen RF. Optimized weighted essentially nonoscillatory schemes for linear waves with discontinuity. Journal of Computational Physics 2001; 174:381-404.

14. Ashcroft G, Zhang X. Optimized prefactored compact scheme. Journal of Computational Physics 2003; 190: 459-477.

15. Popescu M, Shyy W, Garbey M. A study of dispersion-relation-preserving and optimized prefactored compact schemes for wave equation. Journal of Computational Physics 2005; 210(5):705-729.

16. Hu FQ. A Stable. Perfectly matched layer for linearized Euler equations in unsplit physical variables. Journal of Computational Physics 2001; 173:255-480.

17. Hu FQ. Perfectly matched layer absorbing boundary condition for non-linear aeroacoustics. AIAA/CEAS Aeroacoustics Conference, AIAA Paper 2006-2647, Cambridge, Massachusetts, 2006.

18. Freedman A. Acoustic field of a pulsed circular piston. Journal of Sound and Vibration 1994; 170(4):459-519.

19. Lele YS, Hamilton MF. Time-domain modelling of pulsed finite-amplitude sound beams. Journal of Acoustical Society of America 1995; 97(2):906-917.

20. Cleveland RO, Hamilton MF, Blackstock DT. Time-domain modelling of finite-amplitude sound in relaxing fluids. Journal of the Acoustical Society of America 1996; 99(6):3312-3318.

21. Williams EG. Fourier Acoustics Sound Radiation and Nearfield Acoustical Holography. Academic Press: San Diego, CA, 1999.

22. Blackstock DT. Fundamentals of Physical Acoustic. Wiley: New York, 2000. 
23. Tam CKW, Webb JC. Radiation boundary condition and anisotropy correction for finite difference solutions of the Helmholtz equation. Journal of Computational Physics 1994; 113(1):122-133.

24. Yang G, Ingram DM. Cartesian cut-cell method for axisymmetric separating body flows. AIAA Journal 1999; 37(8):905-911.

25. Calhoun D, Le Veque RJ. A Cartesian grid finite-volume method for the advection-diffusion equation in irregular geometries. Journal of Computational Physics 2000; 157:143-180.

26. Ingram DM, Causon DM, Mingham CG. Development in Cartesian cut cell methods. Mathematics and Computers in Simulation 2003; 61:561-572.

27. Udaykumar HS, Mittal R, Shyy W. Computational of solid-liquid phase fronts in the sharp interface limit on fixed grids. Journal of Computational Physics 1999; 153:535-574.

28. Ye T. Direct numerical simulation of a translating vapor bubble with phase change. Ph.D. Dissertation, University of Florida, Department of Mechanical Engineering, Gainesville, FL, 2001.

29. Ye T, Mittal R, Udaykumar HS, Shyy W. An accurate Cartesian grid method for viscous incompressible flows with complex immersed boundaries. Journal of Computational Physics 1999; 156:209-240.

30. Shyy W, Garbey M, Wu J, Appukuttan A. Evaluation of Richardson extrapolation in computational fluid dynamics. Numerical Heat Transfer, Part B 2002; 41:139-164.

31. Shyy W, Garbey M. A least square extrapolation method for improving solution accuracy of PDE computations. Journal of Computational Physics 2003; 186:1-23. 